Article

\title{
Development of a Multi-Criteria Model for Sustainable Reorganization of a Healthcare System in an Emergency Situation Caused by the COVID-19 Pandemic
}

\author{
Dragan Pamučar ${ }^{1,2} \mathbb{D}$, Mališa Žižović ${ }^{3}$, Dragan Marinković ${ }^{4, *}$, Dragan Doljanica ${ }^{5}$, \\ Saša Virijević Jovanović ${ }^{5}\left(\mathbb{D}\right.$ and Pavle Brzaković ${ }^{5}$ \\ 1 Informetrics Research Group, Ton Duc Thang University, Ho Chi Minh City 758307, Vietnam; \\ dragan.pamucar@tdtu.edu.vn \\ 2 Faculty of Civil Engineering, Ton Duc Thang University, Ho Chi Minh City 758307, Vietnam \\ 3 Faculty of Technical Sciences in Cacak, University of Kragujevac, Svetog Save 65, 32102 Cacak, Serbia; \\ zizovic@gmail.com \\ 4 Berlin Institute of Technology, Department of Structural Analysis, 10115 Berlin, Germany \\ 5 MEF Faculty of Applied Management, Economics and Finance, University Business Academy in Novi Sad, \\ Jevrejska 24, 11000 Belgrade, Serbia; dragan.doljanica@mef.edu.rs (D.D.); sasa.virijevic@mef.edu.rs (S.V.J.); \\ pavle.brzakovic@mef.edu.rs (P.B.) \\ * Correspondence: dragan.marinkovic@tu-berlin.de
}

Received: 5 August 2020; Accepted: 10 September 2020; Published: 11 September 2020

check for updates

\begin{abstract}
Healthcare systems worldwide are facing problems in providing health care to patients in a pandemic caused by the SARS-CoV-2 virus (COVID-19). The pandemic causes an extreme disease to spread with fluctuating needs among patients, which significantly affect the capacity and overall performance of healthcare systems. In addition, its impact on the sustainability of the entire economic and social system is enormous and certain sustainable management strategies need to be selected. To meet the challenges of the COVID-19 pandemic and ensure sustainable performance, national healthcare systems must adapt to new circumstances. This paper proposes an original multi-criteria methodology for the sustainable selection of strategic guidelines for the reorganization of a healthcare system under the conditions of the COVID-19 pandemic. The selection of an appropriate strategic guideline is made on the basis of defined criteria and depending on infection capacity and pandemic spread risk. The criteria for the evaluation of strategic guidelines were defined on the basis of a survey in which the medical personnel engaged in the crisis response team during the COVID-19 pandemic in the Republic of Serbia participated. The Level-Based Weight Assessment (LBWA) model and Measuring Attractiveness by a Categorical-Based Evaluation Technique (MACBETH) method were used to determine the weight coefficient criteria, while a novel fuzzy Ranking of Alternatives through Functional Mapping of Criterion Subintervals into a Single Interval (RAFSI) model was used to evaluate the strategic guidelines. The proposed multi-criteria methodology was tested in a case study in the Republic of Serbia. The validity of the proposed methodology is shown through the simulation of changes in input parameters of Bonferroni aggregation functions and through a comparison with other multi-criteria methodologies.
\end{abstract}

Keywords: COVID-19; multi-criteria; level-based weight assessment; fuzzy sets; sustainability

\section{Introduction}

The World Health Organization (WHO) declared a global pandemic, coronavirus disease 2019 (COVID-19) in March 2020, when the international mission of SARS-CoV-2 virus research began. 
The pandemic was caused by a new virus from the coronavirus group, which was first isolated in 1962. Since then, some coronaviruses have been known to infect only some animals, others humans, while some can break the species barrier and cause conditions ranging from mild colds to severe acute respiratory syndrome (SARS). The coronavirus disease (COVID-19) pandemic has brought many surprises and posed many new challenges to healthcare systems worldwide and their sustainable performance. The pandemic has set numerous operational, logistical, organizational and moral-ethical requirements before management, healthcare workers and associates: the organization of specialized training for medical personnel in accordance with emerging conditions; the conversion of facilities for patient accommodation; the procurement of medical equipment; information and communication management; the continuous monitoring of healthcare systems; the availability of health care to specific groups of population in new conditions, etc.

The sustainability of healthcare systems during a pandemic is defined by the Global Health Security Index (GHS Index). The GHS Index assesses the preparedness of healthcare systems to prevent the outbreak and spread of epidemics/pandemics, assesses the preparedness for a rapid response, and also assesses compliance with international protocols in crisis situations, including the COVID-19 pandemic. There are two strategic phases in the fight against the COVID-19 pandemic: (1) Phase I-rapid and aggressive action (rapid reorganization of healthcare systems, restriction of movement, etc.) and (2) Phase II-long-term effort to maintain control over the virus until finding an optimal solution (vaccination, appropriate therapy or increasing the level of community immunity). An integral part of Phase II also includes capacity improvement, forecasting pandemic dynamics, he risk assessment of the further spread of the pandemic and other analyses. The assessment of each parameter reflects structural or other deficiencies in terms of funding, the ability to provide adequate services, management, and workforce and resource management. All of this exposes healthcare systems to great challenges and different outcomes. Due to all of the stated specific characteristics of the COVID-19 pandemic, it is necessary to continuously analyze all weaknesses and strengths of healthcare systems, forming a plan based on adaptive models of healthcare system organization since immediate requirements and the unpredictability of COVID-19 infection require agility and the flexibility of all services to respond in a timely manner, especially qualified medical personnel.

Accordingly, this paper proposes an adaptive model for the organization of a healthcare system in a country, or a region within a country, in cases of emergencies or specific pandemic conditions. The starting point is an assumption that there are (or may be) spas, i.e., spa rehabilitation centers in a given country (region) and that by the interruption of work within the industry, the lives of their users will not be endangered. There are such centers in almost all European countries. In many European countries, spa tourism is very developed, as evidenced by the data shown in Table 1 [1].

Table 1. Overview of spa capacities in several European countries [1].

\begin{tabular}{|c|c|c|c|c|}
\hline No. & Country & Number of Spas & Average Number of Visitors & Average Number of Overnight Stays \\
\hline & Germany & 313 & $>550,000 *$ & $>3,550,000$ \\
\hline & Spain & 128 & $>1,000,000$ & $>2,000,000$ \\
\hline & France & 96 & $>200,000$ & $>4,000,000$ \\
\hline & Austria & 81 & $>500,000$ & $>4,100,000$ \\
\hline & Serbia & 36 & $>200,000$ & $>1,000,000$ \\
\hline & Czech Republic & 34 & $>300,000$ & $>5,500,000$ \\
\hline & Slovakia & 21 & $>100,000$ & $>1,500,000$ \\
\hline & Slovenia & 15 & $>200,000$ & $>1,000,000$ \\
\hline
\end{tabular}

* The average number of visitors and overnight stays is shown for one year.

It should be emphasized that Table 1 shows data for several European countries since we did not have publicly available data for the remaining European countries. Considering these data, we can conclude that in most European countries there is a great potential of spa centers which can give support to healthcare systems in providing health care to patients in a pandemic. 
Hospitals, as healthcare facilities that play a key role in a healthcare system, face problems worldwide in providing health care to patients with different types of diseases. Today, these problems have significantly increased with the occurrence of the COVID-19 pandemic. The pandemic causes an extreme spread of disease with fluctuating needs of patients, which significantly affects the capacity and overall performance of hospitals. In order to cope with the challenges of the COVID-19 pandemic, and remain sustainable under such conditions, national healthcare systems have to adapt to the new circumstances and form special hospitals in which only patients infected with the SARS-CoV-2 virus are treated. This paper proposes a multi-criteria model for the development of strategic guidelines for the adjustment and reorganization of the healthcare system in the Republic of Serbia under the conditions of the COVID-19 pandemic. Four strategic guidelines have been proposed and defined on the basis of a survey conducted with members of the Republic Crisis Response Team in Serbia in the period of March-May 2020. The strategic guidelines are based on the capacity utilization of spa centers and their personnel as a support for the healthcare system of a certain region in the Republic of Serbia. The selection of a strategic guideline that determines the utilization rate of spa centers depends on pandemic infection capacity, which is defined by the WHO. In addition to pandemic infection capacity, the selection of a strategic guideline is also influenced by the prediction of disease spread, which is defined for each country or region individually. Based on pandemic infection capacity and the prediction of disease spread, we determined five levels of risk on the basis of which a multi-criteria model for the selection of strategic guidelines is defined.

Accordingly, the aim of this paper is to develop a multi-criteria model for the evaluation and selection of strategic guidelines that define a strategy for reorganization and adjustment of healthcare systems under the conditions of the COVID-19 pandemic. The proposed multi-criteria model is based on the application of the Level-Based Weight Assessment (LBWA) model [2], Measuring Attractiveness by a Categorical-Based Evaluation Technique (MACBETH) method [3] and the Ranking of Alternatives through Functional Mapping of Criterion Subintervals into a Single Interval (RAFSI) model [4]. The LBWA-MACBETH model was used to determine the weight coefficients of criteria, while the fuzzy RAFSI model was used to evaluate and select strategic guidelines for reorganizing and adjusting the healthcare system. In addition, the proposed model has the following advantages which improve the literature that considers the application of multi-criteria techniques in the medical field:

- The original multi-criteria methodology was developed for the reorganization and adjustment of healthcare systems in an emergency situation caused by the COVID-19 pandemic;

- The multi-criteria methodology allows the evaluation and selection of a strategy for the reorganization of a healthcare system of a region/country depending on a risk level defined on the basis of pandemic infection capacity and the prediction of disease spread;

- Strategic guidelines and criteria for the evaluation of guidelines were defined on the basis of several months of research in which medical experts and other members of the crisis response team in Serbia participated;

- The presented multi-criteria methodology represents a contribution to the decision-making methodology, which is reflected through an original extension of the RAFSI algorithm in a fuzzy environment;

- Since decision making under the conditions of the COVID-19 pandemic is accompanied by numerous uncertainties and dynamic characteristics of input parameters, a special contribution of this paper is an original hybrid fuzzy LBWA-MACBETH-RAFSI model that allows the evaluation and selection of alternative solutions under conditions of uncertainty;

- The fuzzy LBWA-MACBETH-RAFSI multi-criteria methodology is characterized by flexibility and applicability in various areas where strategic decision making is required;

- The proposed multi-criteria methodology provides a new framework for resource management. The model presented in this paper can help decision makers in hospitals and national policy 
makers to establish strategic guidelines for the adjustment of healthcare systems depending on the capacity of the COVID-19 pandemic.

The paper is structured into four sections. After the introduction, in which the problem and objectives of the paper are presented, the second section of the paper presents an analysis of the literature that includes studies which consider the application of multi-criteria techniques in the healthcare field. In the third section of the paper, a multi-criteria model for the evaluation of strategic guidelines for the organization of a healthcare system under the conditions of the COVID-19 pandemic is formulated. The fourth section presents the implementation of the multi-criteria model and the validation of the results shown. In the fourth section of the paper, conclusions and guidelines for future research are presented.

\section{Literature Review}

The assessment of preparedness of healthcare systems to work in crisis situations, such as the COVID-19 pandemic, has been performed in a small number of papers to date. This especially refers to the application of multi-criteria optimization techniques for assessing the degree of organization of healthcare systems, as well as for making strategic decisions. The analysis of the literature shows that most authors apply two main concepts for the analysis of the preparedness of healthcare systems in crisis situations. The first concept implies the application of multi-criteria models, while the second concept is based on the application of statistical tools for data processing and the analysis of their statistical correlation. In their study, Nekoie-Moghadam et al. [5] presented a comprehensive literature review with an analysis of various methodologies used to assess the ability of healthcare institutions to work in crisis situations. Nekoie-Moghadam et al. [5] considered studies that analyzed the level of preparedness of healthcare institutions in terms of logistical capacity, capacity of medical personnel, preparedness of communications for an emergency, management skills, training of medical and non-medical personnel, development of procedures for evacuation of seriously ill people and isolation in case of infectious diseases, disaster recovery, transport capacity, etc. [5-7]. In all analyzed studies, the authors applied different statistical tools for processing data collected by questionnaire surveys [5]. A comprehensive review of the literature that considers the application of statistical tools for the analysis of the preparedness of healthcare institutions to work in crisis situations is also presented in [5-8]. Tabatabaei and Abbasi [9] defined the hospital safety index on the basis of which the risk assessment of healthcare institutions for work in crisis conditions was performed. In their study, Samsuddin et al. [10] showed that human resources, their training and the ability of healthcare institution to adjust in a timely manner are key factors for defining a risk level for work in crisis situations. Marzaleh et al. [11] performed an analysis of the assessment of the preparedness of emergency services in hospitals to work in crisis situations. For the evaluation, they used 31 criteria which were processed using statistical tools and the Delphi technique. A similar methodology was applied by Shabanikiya et al. [12] for hospital capacity analysis. Mulyasari et al. [13] assessed hospital capacity preparedness in Japan using six parameters that consisted of 21 indicators. The study analyzed the preparedness of healthcare systems to work in a crisis situation caused by a tsunami in the Tohoku area.

In addition to the application of statistical tools for assessing the significance of factors for the evaluation of healthcare institutions, there are a number of publications in which the evaluation of healthcare institutions is performed using multi-criteria tools. Hosseini et al. [14] evaluated hospitals using the Technique for Order of Preference by Similarity to Ideal Solution (TOPSIS) multi-criteria technique. The significance of four clusters and 21 factors used to evaluate healthcare facilities was defined based on experts' group preferences. In their study, Ortiz-Barrios et al. [15] demonstrated the application of a complex hybrid multi-criteria technique for the evaluation of hospitals. An integrated Analytic Hierarchy Process (AHP) and Decision-Making Trial and Evaluation Laboratory (DEMATEL) model were used to determine the weight coefficients of factors, while the Technique for Order of Preference by Similarity to Ideal Solution method was used for the evaluation. In order to consider interrelations and define graphically the relationships between the factors used for evaluation, 
Ortiz-Barrios et al. [15] used the Decision-Making Trial and Evaluation Laboratory (DEMATEL) method. After defining the significance and interrelation of factors using the DEMATEL method, Ortiz-Barrios et al. [15] showed the TOPSIS methodology as a powerful tool for defining ideal reference values on the basis of which the comparison and evaluation of hospitals were performed. Similar to [15], the advantages of using the DEMATEL technique in their study were shown by Roy et al. [16]. However, in contrast to previous studies that ignored the existence of inaccuracies and uncertainties in the data used to evaluate healthcare systems, in their study, Roy et al. [16] used rough numbers to present inaccuracies in expert preferences.

Given that the COVID-19 pandemic is ongoing and that there are no publications considering the problem of evaluation and assessment of the preparedness of medical institutions to work under the conditions of COVID-19 pandemic, the following section analyzes only a few articles dealing with multi-criteria techniques in the COVID-19 pandemic. Sarkar [17] performed a spatial analysis and mapping of areas susceptible to COVID-19 infection in Bangladesh. A multi-criteria methodology (AHP method), which was implemented in a Geographic Information System (GIS), was used to map the area. In addition to the presented study, a spatial analysis of the risk of spreading COVID-19 infection in urban areas in Italy was performed by Sangiorgio and Parisi [18]. They used the Artificial Neural Network and GIS to create spatial risk maps in 257 city districts. Nardo et al. [19] showed the application of multi-criteria decision analysis to determine weights for eleven criteria in order to prioritize COVID-19 non-critical patients for admission to hospital in healthcare settings with limited resources. Yildirim et al. [20] evaluated the available COVID-19 treatment options in hospitals. For the evaluation, they used a hybrid methodology based on the application of two well-known multi-criteria tools: (1) Preference Ranking Organization Method for Enrichment of Evaluations (PROMETHEE) and (2) Vlsehriterijumska Optimizacija I Kompromisno Resenje (VIKOR) method. The mathematical formulation of both models is presented by fuzzy arithmetic and fuzzy sets.

By analyzing the presented articles, we can conclude that there is a gap in the literature that considers the application of hybrid multi-criteria methods for the evaluation of healthcare systems under the conditions of the COVID-19 pandemic. In general, there are only a few articles in the literature that consider the application of hybrid multi-criteria techniques for the evaluation of hospital capacities in crisis situations $[14,15,21]$. Despite the fact that decision making under the conditions of the COVID-19 pandemic and crisis situations in general is accompanied by a greater or lesser number of uncertainties, there are only a few studies that apply fuzzy numbers to treat uncertainties [20]. Additionally, the analysis of the literature shows that there is no study that considers the possibility of including spa centers in healthcare systems in order to reduce the pressure on primary hospital capacities under the conditions of COVID-19 pandemic. This study proposes an original multi-criteria methodology (fuzzy LBWA-MACBETH-RAFSI model) that has not been considered in the literature so far. The proposed methodology fills a gap in the literature considering the application of multi-criteria techniques for evaluating healthcare systems under the conditions of COVID-19 pandemic and for evaluation of healthcare systems in general. It should be emphasized that the novel fuzzy LBWA-MACBETH-RAFSI multi-criteria methodology has a universal algorithm that can be applied to solve various real-world problems, which refreshes and improves the field of multi-criteria decision making from a theoretical and methodological perspective.

\section{MCDM Methodology for Proposing a Possible Organization of a Healthcare System under the Conditions of the COVID-19 Pandemic}

The basic model of organizing healthcare systems in case of emergency is that each healthcare center within a region/country organizes work within its infectious disease ward in cooperation with appropriate infectious disease clinics. Experiences from a period of February-May 2020 have shown that organizing a healthcare system in this way satisfies the needs of users only in the case of low to medium pandemic infection capacity, i.e., in the case of low to medium disease spread risk. In the case of a higher pandemic infection capacity and higher pandemic spread risk, it is necessary to 
reorganize the healthcare system using secondary healthcare facilities. A large number of countries, including Serbia, in addition to secondary healthcare facilities, have used other facilities of large capacity (e.g., sports halls) for the accommodation and isolation of mildly ill patients.

However, in case of an emergency, spa centers can be successfully re-oriented to providing care for patients referred by appropriate healthcare institutions. Depending on the infection capacity and pandemic spread risk, it is possible to define variants of the inclusion of spa centers for patient care. Each variant of the inclusion of spa centers in healthcare systems is a strategic guideline for a possible reorganization of healthcare systems. In this research, four variants (strategic guidelines) for the reorganization of healthcare systems are defined:

Strategic guideline $1(A 1)$ : A healthcare center organizes its work through three levels. The initial level implies that each healthcare center organizes the admission of infected patients within its infectious disease ward in cooperation with infectious disease clinics. When the healthcare center capacity becomes insufficient for the admission and care of patients, the second level of organization is applied. Within the second level, the healthcare center is divided into the main and infectious disease part of the healthcare center. In the infectious disease part, patients who have received positive results after testing for the coronavirus are taken care of. The main part of the healthcare center provides medical services to patients who are not infected. If the second level of organization cannot provide a satisfactory level of health care, the third level of organization is applied. In the third level, the organization of the healthcare center of the second level is maintained, activating additional capacities for the admission of infected patients, such as sports halls, hotels, hostels, etc., which are adapted for the admission and care of positive patients.

Strategic guideline 2 (A2): A healthcare center organizes its work through two levels. The initial level, as for $A 1$, implies that the healthcare center organizes the admission of infected patients within its infectious disease ward in cooperation with infectious disease clinics. When the healthcare center capacity becomes insufficient for the admission and care of positive patients, the second level of the organization is applied. The second level of organization involves the activation of spa centers to provide medical services to patients who are positive to the coronavirus and have milder symptoms. The infectious disease ward of the healthcare center treats patients who are positive to the coronavirus, who have more severe symptoms and who need artificial lung ventilation. Other units (non-infectious disease units) of the healthcare center provide medical services to patients suffering from other diseases. Here, a spa center represents a recreation and wellness center that is not connected to any healthcare center, but which has staff that as medical personnel can be involved in providing care for positive patients. Patients from superior healthcare institutions are referred to the spa center. In accordance with their protocols, healthcare institutions engage medical personnel to work in spa centers. The defined protocols also apply to the present staff of the spa center, which may, depending on the assessment of the crisis response team, be engaged in treatment.

Strategic guideline $3(A 3)$ : A healthcare center organizes its work through two levels: (1) the initial level implies that the healthcare center organizes the admission of infected patients within its infectious disease ward in cooperation with infectious disease clinics and (2) the second level is applied when the healthcare center capacity becomes insufficient for the admission and care of positive patients. The second level involves the use of spa centers to provide medical services to patients infected with the coronavirus. In this strategic guideline, spa centers are rehabilitation centers that are an integral part of the observed healthcare center. In addition to medical personnel from superior healthcare institutions, the personnel from the spa center are directly involved in a work process. For the staff from the spa center, an assessment is made as to whether they are trained to work in emergency situations. For non-trained staff of rehabilitation center, the healthcare center organizes training for work in pandemic conditions.

Strategic guideline 4 (A4): The strategic guideline $A 4$ represents healthcare center organization through two levels. Within the initial level, the healthcare center organizes the admission and care of all patients. Coronavirus-positive patients are treated within an infectious disease ward in collaboration 
with infectious disease clinics. Patients who are not positive are treated in the remaining specialized units of the healthcare center. When the healthcare center capacity becomes insufficient for the admission and care of patients, the second level of organization is applied, which includes the use of spa centers. Here, a spa center represents a rehabilitation center that is connected to several healthcare centers that gravitate towards the spa center and it is capable of providing medical care for patients suffering from infectious diseases. The personnel of this center are trained to work under the conditions of a pandemic. In addition to medical personnel from a directly superior healthcare center and trained staff of the spa center, the personnel of other regional centers that gravitate towards the spa center are also involved in a work process of the spa center. At the same time, these regional centers refer their patients for treatment to the spa center. If the spa center has reached its full capacity, other facilities, such as halls, hotels, hostels and others, are used and adapted for the admission and care of positive patients.

The selection of an appropriate strategic guideline for the reorganization of healthcare systems is made depending on infection capacity and pandemic spread risk. The main cause of the epidemic is that one infected person spreads the infection to (on average) $p$ other persons. If $p>1$, the infection spreads exponentially; if $p<1$, the infection subsides and disappears [22]. The parameter $p$ can be changed in several ways. One way is to find a number of immune people in the community over time that cannot be infected, which reduces $p$. The higher the number of immune people, the lower the $p$, and when $p$ falls below 1 , the infection subsides [23]. Based on the value of $p$ and risk assessment $(\theta)$, infection capacity and pandemic spread risk $(\delta), \delta=p \cdot \theta$, are defined. The value of $\theta$ is defined by experts in the crisis response team on the basis of a five-degree scale: 1 -very low risk, 2-low risk, 3-medium risk, 4-high risk and 5-very high risk. For the purpose of this study, we defined a scale on the basis of which infection capacity and pandemic spread risk $(\delta)$ are determined; see Table 2. The limit values of the scale are defined on the basis of recommendations by experts from the crisis response team in the Republic of Serbia.

Table 2. A scale for defining infection capacity and pandemic spread risk.

\begin{tabular}{ccc}
\hline No. & Linguistic Value & Numerical \\
\hline 1. & Low infection capacity and risk & $0 \leq \delta \leq 2.0$ \\
2. & Medium infection capacity and risk & $2.0<\delta \leq 3.0$ \\
3. & Serious infection capacity and risk & $3.0<\delta \leq 5.0$ \\
4. & High infection capacity and risk & $5.0<\delta \leq 7.0$ \\
5. & Very high infection capacity and risk & $>7.0$ \\
\hline
\end{tabular}

After defining infection capacity and pandemic spread risk, a multi-criteria model is applied to select an appropriate strategic guideline for the reorganization of the healthcare system; see Figure 1 .

The multi-criteria model implies the application of the LBWA model for determining the weight coefficients of criteria. The evaluation and selection of an appropriate guideline is completed using the fuzzy RAFSI method. The evaluation of guidelines (alternatives) in relation to defined criteria $C_{j}$ $(j=1,2, \ldots, n)$ is performed by experts using a predefined fuzzy linguistic scale. After defining expert correspondent matrices, expert preferences are aggregated into an averaged initial matrix in which uncertainties are represented by triangular fuzzy numbers. Fuzzy linguistic variables were chosen due to their ability to present uncertainties and dilemmas that exist when evaluating strategic guidelines. In the following sections, the mathematical formulation of the fuzzy LBWA-MACBETH-RAFSI multi-criteria methodology is given. 


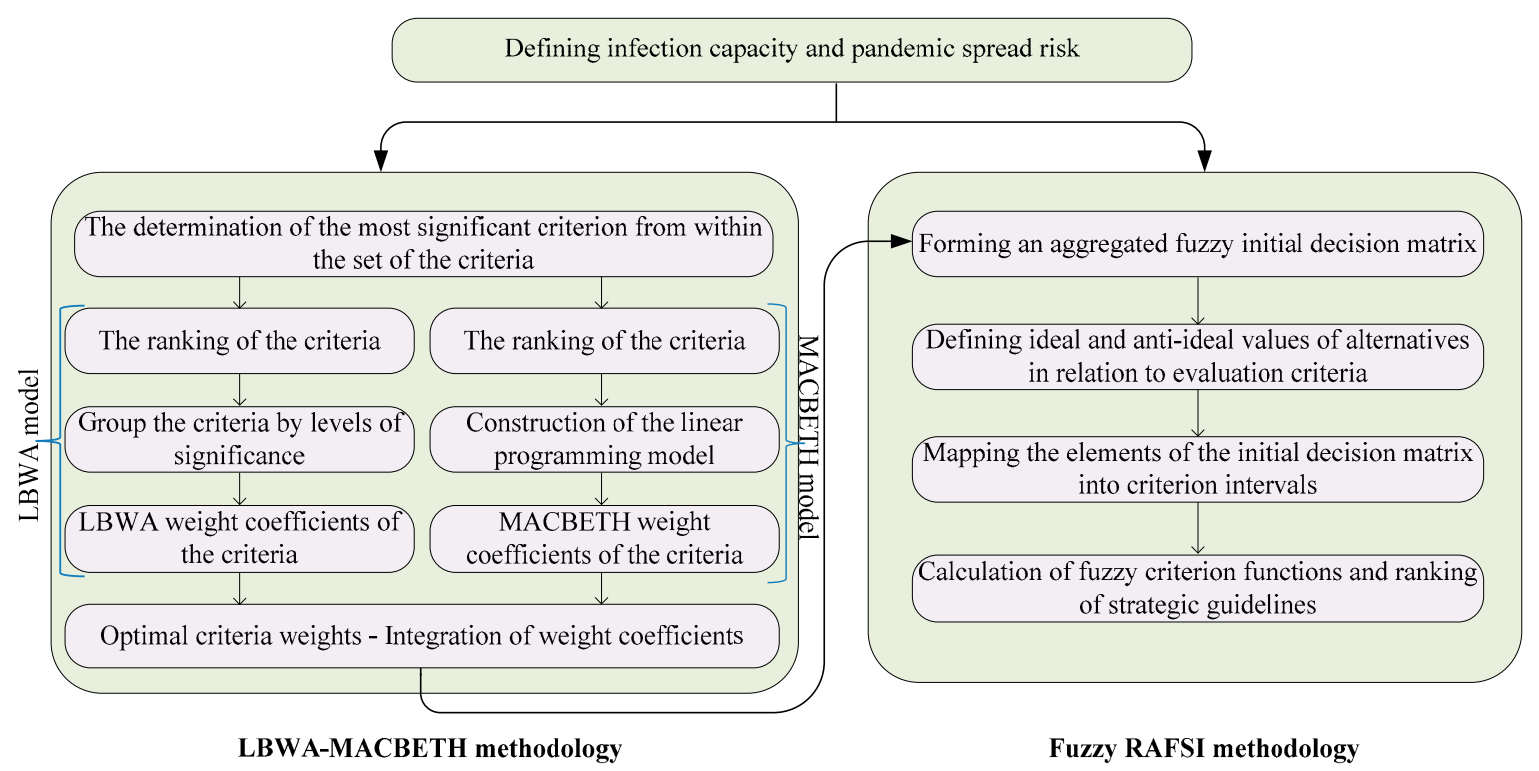

Figure 1. Multi-criteria model for the selection of strategic guidelines for the reorganization of the healthcare system.

\subsection{LBWA Model for Determining Weight Coefficients of Criteria}

The LBWA method (Figure 2) for determining weight coefficients of criteria is characterized by a small number of pairwise comparisons of criteria and a rational and logical mathematical algorithm [2]. Despite the fact that the LBWA method belongs to a group of multi-criteria methods developed recently, there are already a number of publications that exploit the advantages of the LBWA methodology [24-28].

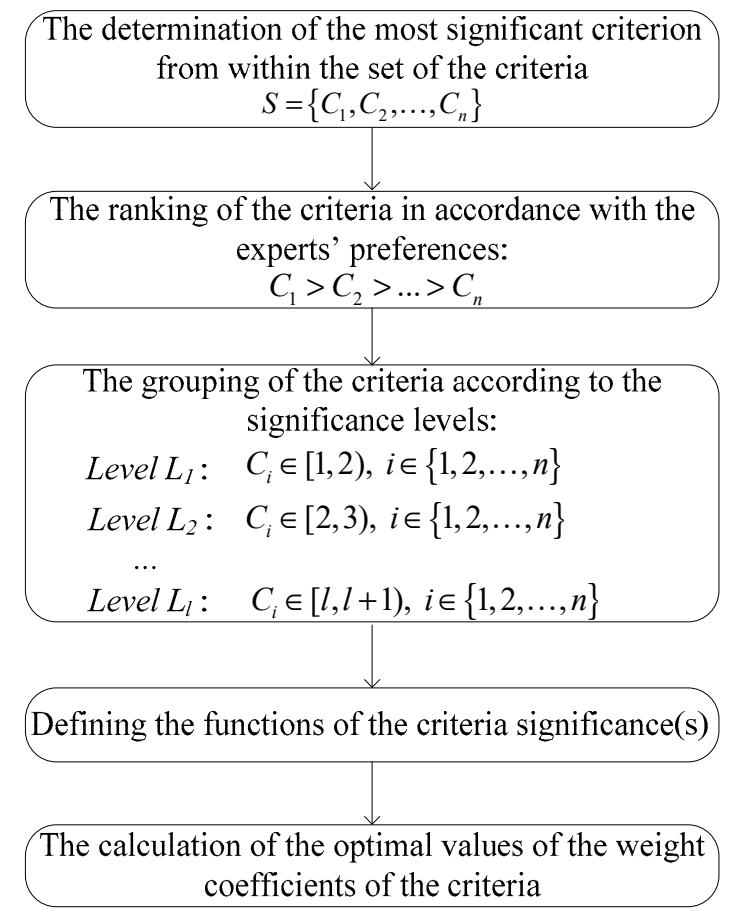

Figure 2. The LBWA model.

From a group of subjective models for determining the weights of criteria, the LBWA methodology is distinguished on the basis of the following advantages: (1) The LBWA model allows the calculation of weight coefficients with a small number of criteria comparisons; (2) The LBWA model algorithm does 
not become more complex with the increase in the number of criteria, which makes it suitable for use in complex multi-criteria decision making (MCDM) models with a larger number of evaluation criteria; (3) The LBWA model allows decision makers to present their preferences through a logical algorithm when prioritizing criteria; (4) LBWA methodology eliminates inconsistencies in expert preferences since comparisons in the LBWA model are always consistent. Keeping in mind the stated advantages of LBWA, the authors decided to use the LBWA model for determining the weights of the criteria in this paper. The following section presents the algorithm for the LBWA model that includes six steps.

Step 1: Determining the most significant criterion from the set of criteria. Assume that there is a group of $k$ experts who have defined the set of criteria $C=\left\{C_{1}, C_{2}, \ldots, C_{n}\right\}$, where $n$ represents the total number of criteria. Experts arbitrarily make a decision on the selection of the most influential criterion $C_{B}$ from the set $C$.

Step 2: Grouping criteria by levels of significance. The following algorithm is used to group the criteria by levels of significance:

Level $L_{1}$ : At the level $L_{1}$, group the criteria whose significance is equal to the significance of the criterion $C_{B}$ or up to twice less than the significance of the criterion $C_{B}$, i.e., $C_{j} \in[1,2), j=1,2, \ldots, n$.

Level $L_{2}$ : At the level $L_{2}$, group the criteria whose significance is exactly twice less than the significance of the criterion $C_{B}$ or up to three times less than the significance of the criterion $C_{B}$, i.e., $C_{j} \in[2,3), j=1,2, \ldots, n$.

Level $L_{l}$ : At the level $L_{l}$, group the criteria whose significance is exactly $l$ times less than the significance of the criterion $C_{B}$ or up to $l+1$ times less than the significance of the criterion $C_{B}$, i.e., $C_{j} \in[l, l+1), j=1,2, \ldots, n$.

If the significance of the criterion $C_{j}$ is denoted by $s\left(C_{j}\right)$, where $j=1,2, \ldots, n$, then we have $L=L_{1} \cup L_{2} \cup \cdots \cup L_{l}$, where for every level $i=1,2, \ldots, l$, the following applies:

$$
L_{i}=\left\{C_{i_{1}}, C_{i_{2}}, \ldots, C_{i_{s}}\right\}=\left\{C_{j} \in L: i \leq s\left(C_{j}\right)<i+1\right\}
$$

Step 3: Comparison of criteria by significance. Within the formed levels of significance, each expert compares the criteria by their significance. Each criterion $C_{i_{p}} \in L_{i}$ within the subset $C_{i}=\left\{C_{i_{1}}, C_{i_{2}}, \ldots, C_{i_{s}}\right\}$ is assigned the value $I_{i_{p}}$ such that the most significant criterion $C_{B}$ within the first level is assigned the value $I_{B}=0$. Moreover, if $C_{i_{p}}$ is more significant than $C_{i_{q}}$ then $I_{i_{p}}<I_{i_{q}}$, and if $C_{i_{p}}$ is equivalent to $C_{i_{q}}$ then $I_{i_{p}}=I_{i_{q}}$. The maximum value on the scale for the comparison of criteria is defined by applying Equation (2):

$$
\lambda=\max \left\{\left|L_{1}\right|,\left|L_{2}\right|, \ldots,\left|L_{l}\right|\right\}
$$

Step 4: Defining the elasticity coefficient. Based on the defined maximum value of the scale for the comparison of criteria $(\lambda)$, Equation (2), the elasticity coefficient $\Delta \lambda(\Delta \lambda>\lambda)$ is defined:

$$
\Delta \lambda=\max \left\{\left|L_{1}\right|,\left|L_{2}\right|, \ldots,\left|L_{l}\right|\right\}
$$

Step 5. Calculation of the influence function of criteria. For each criterion $C_{j}$ within the observed level of significance $C_{j} \in L_{i}$, the influence function of the criterion is defined:

$$
f\left(C_{j}\right)=\frac{\Delta \lambda}{i \cdot \Delta \lambda+I_{i_{p}}}
$$

where $i$ represents the number of significance level in which the criterion $C_{j}$ is classified, $\Delta \lambda$ represents the elasticity coefficient, while $I_{i_{p}}$ represents the value assigned to the criterion $C_{j}$ within the observed level $L_{i}$. 
Step 6. Calculation of the optimal values of the weight coefficients of criteria. By applying Equation (5), the weight coefficient of the most influential criterion $C_{B}$ is calculated:

$$
w_{B}^{L B W A}=\frac{1}{1+\sum_{\substack{j=1 \\ j \neq B}}^{n} f\left(C_{j}\right)}
$$

where $w_{B}$ represents the value of the weight coefficient of the most influential criterion $\left(C_{B}\right)$, while $f\left(C_{j}\right)$ represents the function of the influence of criteria defined by Equation (4).

The values of the weight coefficients of the remaining criteria are obtained by applying Equation (6):

$$
w_{j}^{L B W A}=f\left(C_{j}\right) \cdot w_{B}
$$

where $w_{B}$ represents the value of the weight coefficient of the most influential criterion $\left(C_{B}\right)$.

\subsection{MACBETH Model for Determining Weight Coefficients of Criteria}

The Measuring Attractiveness by a Categorical-Based Evaluation Technique (MACBETH) method [3] is one of the multi-criteria decision making methods that depends upon the qualitative judgments between alternatives and criteria [3]. The algorithm of the MACBETH method is similar to the algorithm of the Analytical Hierarchy Process (AHP) method, since both methods use comparative comparison matrices. The main difference between these two methods is that AHP uses a ratio scale but MACBETH uses an interval scale [3]. The semantic categories used for pairwise comparisons can be seen in Table 3.

Table 3. Semantic Categories for Measuring Attractiveness by a Categorical-Based Evaluation Technique (MACBETH).

\begin{tabular}{cc}
\hline Semantic Categories & Scale \\
\hline No & 0 \\
Very weak & 1 \\
Weak & 2 \\
Moderate & 3 \\
Strong & 4 \\
Very strong & 5 \\
Extreme & 6 \\
\hline
\end{tabular}

The MACBETH method uses a linear programming model for finding the importance levels of the alternatives or criteria. In this study, the MACBETH method is used for the importance levels of the criteria. This linear programming model has been constructed as follows (Burgazoğlu, 2015):

$$
\begin{aligned}
& z_{\min }=\phi\left(o_{1}\right) \\
& o_{i}>o_{j} \Rightarrow \phi\left(o_{i}\right) \geq \phi\left(o_{j}\right)+\delta(i, j) ; \forall o_{i}, o_{j}, i, j \in\{1,2, \ldots, n\} \\
& \phi\left(o_{i}\right)-\phi\left(o_{j}\right) \geq \phi\left(o_{k}\right)-\phi\left(o_{l}\right)+\delta(i, j, k, l) ; \forall o_{i}, o_{j}, i, j, k, l \in\{1,2, \ldots, n\} \\
& \phi\left(o_{n}\right)=1
\end{aligned}
$$

After solving linear model (7), the criteria weights have been determined. The next step is to normalize these values. The normalization process can be made as in Equation (8).

$$
w_{j}^{M A C B E T H}=\frac{\phi\left(o_{j}\right)}{\sum_{j=1}^{n} \phi\left(o_{j}\right)}
$$


The final values of the weighting coefficients of the criteria were obtained by fusing the values of the weighting coefficients obtained by the LBWA and MACBETH methodology, Equation (9):

$$
w_{j}=\xi \cdot w_{j}^{L B W A}+(1-\xi) \cdot w_{j}^{M A C B E T H}
$$

where $w_{j}(j=1,2, \ldots, n)$ represents the final values of the weighting coefficients, $w_{j}^{L B W A}$ represents the value of the weighting coefficient obtained using the LBWA methodology, $w_{j}^{M A C B E T H}$ represents the value of the weighting coefficient obtained using the MACBETH methodology, while the coefficient $\xi \in[0,1]$ defines the percentage share of weights criteria in the final decision. It is recommended to define a value $\xi=0.5$ for the initial ranking of alternatives, since for this value both methodologies equally ( $50 \%$ each) participate in defining the final weights of the criteria.

\subsection{Fuzzy RAFSI Method for the Evaluation of Alternatives}

The Ranking of Alternatives through Functional Mapping of Criterion Subintervals into a Single Interval (RAFSI) method [4] integrates three starting points on which it bases its decision making algorithm: (1) defining referential points-ideal and anti-ideal criteria values; (2) defining the relationship between alternatives in relation to defined ideal/anti-ideal values; (3) defining criterion functions that map criterion sub-intervals into a unique criterion interval. The RAFSI method has three key advantages that recommend it for further application [4]: (i) the simple algorithm of the RAFSI method; (ii) the fact that the RAFSI method has a new methodology for data standardization that enables the transformation of data from an initial decision matrix into an interval that is suitable for rational decision making; (iii) the fact that the mathematical formulation of the RAFSI method eliminates the rank reversal problem, which is one of the significant shortcomings of many traditional MCDM methods.

The following section shows the extension of the RAFSI method to a fuzzy environment (RAFSI-F). By applying fuzzy sets, the RAFSI algorithm has been improved and adapted to handle inaccuracies and uncertainties that arise when solving real-world problems. The algorithm of the RAFSI-F method is realized through six steps:

Step 1: Forming an aggregated fuzzy initial decision matrix. Assume that the evaluation of alternatives from the set $A_{i}(i=1,2, \ldots, m)$ is carried out by $k$ experts. The experts evaluate the alternatives in relation to the defined set of criteria $C_{j}(j=1,2, \ldots, n)$ using a predefined fuzzy linguistic scale. Then, we can present the judgment of $k$ expert as a matrix $X^{(e)}=\left[\widetilde{\xi}_{i j}^{(e)}\right]_{m \times n^{\prime}}$, where $1 \leq e \leq k$.

$$
X^{(e)}=\left[\begin{array}{cccc}
\widetilde{\xi}_{i j}^{(e)} & \widetilde{\xi}_{12}^{(e)} & \cdots & \widetilde{\xi}_{1 n}^{(e)} \\
\widetilde{\xi}_{21}^{(e)} & \widetilde{\xi}_{22}^{(e)} & \cdots & \widetilde{\xi}_{2 n}^{(e)} \\
\vdots & \vdots & \ddots & \vdots \\
\widetilde{\xi}_{m 1}^{(e)} & \widetilde{\xi}_{m 2}^{(e)} & \cdots & \widetilde{\xi}_{m n}^{(e)}
\end{array}\right]_{m \times n} \quad ; 1 \leq i \leq m ; 1 \leq j \leq n ; 1 \leq e \leq k
$$

where $\widetilde{\xi}_{i j}^{(e)}=\left(\xi_{i j}^{l(e)}, \xi_{i j}^{s(e)}, \xi_{i j}^{u(e)}\right) ;(i=1, \ldots, m ; j=1, \ldots, n)$ represents a fuzzy value from the fuzzy linguistic scale.

Accordingly, we obtain a matrix $X^{(e)}$ for each expert, i.e., we obtain $k$ expert initial decision matrices $X^{(1)}, X^{(2)}, \ldots, X^{(e)}, \ldots, X^{(k)}$. For each expert matrix $X^{(e)}=\left[\widetilde{\xi}_{i j}^{(e)}\right]_{m \times n}$ at position $(i, j)$, we obtain a fuzzy sequence $\widetilde{\xi}_{i j}^{(e)}=\left(\xi_{i j}^{l(e)}, \xi_{i j}^{s^{(e)}}, \xi_{i j}^{u(e)}\right)$. By applying Equation (11), we obtain an averaged fuzzy number 
$\widetilde{\xi}_{i j}=\left(\xi_{i j^{\prime}}^{l} \xi_{i j^{\prime}}^{s} \xi_{i j}^{u}\right)$, where $\xi_{i j}^{l}$ and $\xi_{i j}^{u}$ represent the lowest and highest limits of the fuzzy number interval, respectively, while $\xi_{i j}^{s}$ represents the value in which the fuzzy number $\widetilde{\xi}_{i j}$ has a maximum value.

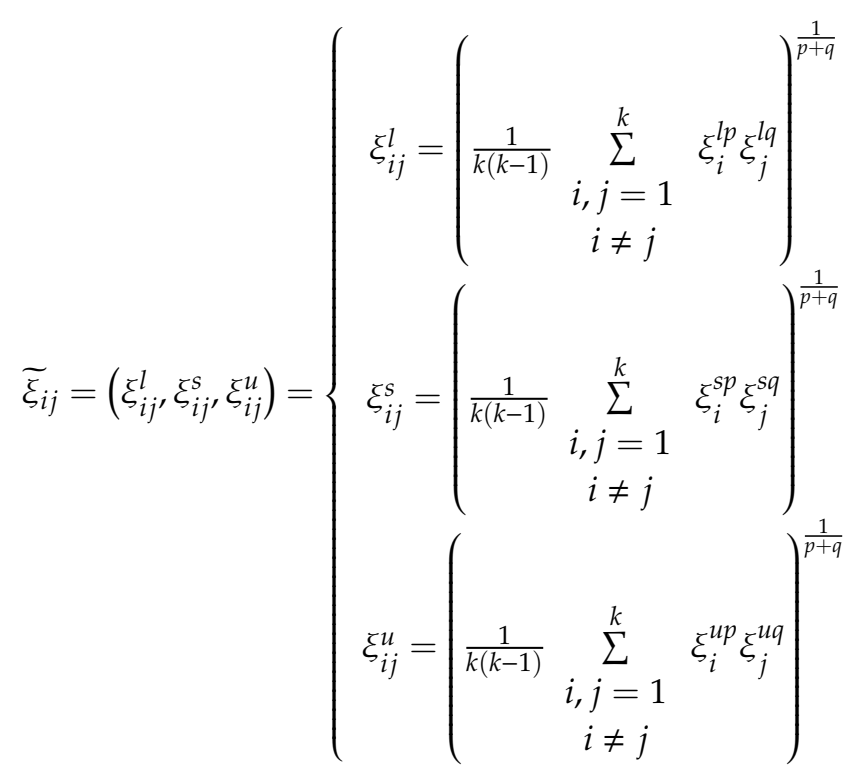

By applying Equation (11), we obtain an averaged fuzzy initial decision matrix

$$
X=\left[\begin{array}{cccc}
\widetilde{\xi}_{11} & \widetilde{\xi}_{12} & \cdots & \widetilde{\xi}_{1 n} \\
\widetilde{\xi}_{21} & \widetilde{\xi}_{22} & \cdots & \widetilde{\xi}_{2 n} \\
\vdots & \vdots & \ddots & \vdots \\
\widetilde{\xi}_{m 1} & \widetilde{\xi}_{m 2} & \cdots & \widetilde{\xi}_{m n}
\end{array}\right]_{m \times n}
$$

where $\widetilde{\xi}_{i j}=\left(\xi_{i j}^{l}, \xi_{i j}^{s}, \xi_{i j}^{u}\right)$ denotes the value of the $i$-th alternative for the $j$-th criterion $(i=1,2, \ldots, m$; $j=1,2, \ldots, n)$. The elements of the matrix $X$ (Equation (12)) represent the fuzzy numbers obtained by averaging the elements from the expert initial decision matrices $X^{(k)}$.

Step 2: Defining ideal and anti-ideal values. For each criterion $C_{j}(j=1,2, \ldots, n)$, a decision maker defines $\widetilde{\xi}_{I_{j}}$ and $\widetilde{\xi}_{N_{j}}$, where $\widetilde{\xi}_{I_{j}}$ represents an ideal value by criterion $C_{j}$, while $\widetilde{\xi}_{N_{j}}$ represents an anti-ideal value by criterion $C_{j}$.

Step 3: Mapping the elements of the initial decision matrix into criterion intervals. In the previous step, the criterion intervals $C_{j} \in\left[\widetilde{\xi}_{N_{j}}, \widetilde{\xi}_{I_{j}}\right]$ (for max criteria) and $C_{j} \in\left[\widetilde{\xi}_{I_{j}}, \widetilde{\xi}_{N_{j}}\right]$ (for min criteria) are defined.

For each alternative from the set $A_{i}(i=1,2, \ldots, m)$, we define the function $\widetilde{f_{A_{i}}}\left(C_{j}\right)$ that maps the criterion intervals from the aggregated initial decision matrix (12) to the criterion interval $\left[n_{1}, n_{b}\right]$, Equation (13):

$$
\widetilde{f}_{A_{i}}\left(C_{j}\right)=\frac{n_{b}-n_{1}}{\widetilde{\xi}_{I_{j}}-\widetilde{\xi}_{N_{j}}} \widetilde{\xi}_{i j}+\frac{\widetilde{\xi}_{I_{j}} \cdot n_{1}-\widetilde{\xi}_{N_{j}} \cdot n_{b}}{\widetilde{\xi}_{I_{j}}-\widetilde{\xi}_{N_{j}}}
$$

where $n_{b}$ and $n_{1}$ represent a relation that shows how much an ideal value is better than anti-ideal value; $\widetilde{\xi}_{I_{j}}$ and $\widetilde{\xi}_{N_{j}}$ represent the ideal and anti-ideal value for the criterion $C_{j}$, respectively, while $\widetilde{\xi}_{i j}$ denotes the value of the $i$-th alternative for the $j$-th criterion from the aggregated initial decision matrix (12). It is recommended that the ideal value is at least six times better than the anti-ideal (barely acceptable values), i.e., that $n_{1}=1$ and $n_{b}=6$. 
Thus, we obtain a standardized decision matrix $T=\left[\widetilde{\varphi}_{i j}\right]_{m \times n}(i=1,2, \ldots, m, j=1,2, \ldots, n)$ in which all elements of the matrix are transformed into an interval $\bar{\varphi}_{i j} \in\left[n_{1}, n_{b}\right]$.

$$
T=\begin{aligned}
& C_{1} \\
& A_{2} \\
& \vdots \\
& A_{m}
\end{aligned}\left[\begin{array}{lllll}
\widetilde{\varphi}_{11} & \widetilde{\varphi}_{12} & \ldots & C_{n} \\
\widetilde{\varphi}_{21} & \widetilde{\varphi}_{22} & \ldots & \widetilde{\varphi}_{1 n} \\
\vdots & \vdots & \ddots & \vdots \\
\widetilde{\varphi}_{m 1} & \widetilde{\varphi}_{m 2} & \cdots & \widetilde{\varphi}_{m n}
\end{array}\right]
$$

where the elements $\widetilde{\varphi}_{i j}$ of the matrix $T$ are obtained by applying Equation (13), i.e., $\widetilde{\varphi}_{i j}=f_{A_{i}}\left(C_{j}\right)$.

Step 4: Forming a normalized decision matrix $N=\left[\hat{\varphi}_{i j}\right]_{m \times n}(i=1,2, \ldots, m, j=1,2, \ldots, n)$. By applying Equation (15), the normalization of the element of matrix $T$ is performed.

$$
\hat{\varphi}_{i j}=\left\{\begin{array}{l}
\frac{\widetilde{\varphi}_{i j}}{2 A}, \text { for max criteria } \\
\frac{H}{2 \widetilde{\varphi}_{i j}}, \text { for min criteria }
\end{array}\right.
$$

where $A$ and $H$ represent the arithmetic and harmonic means of the elements $n_{1}$ and $n_{b}$, respectively, i.e.,

$$
\begin{aligned}
& A=\frac{n_{1}+n_{b}}{2} \\
& H=\frac{2}{\frac{1}{n_{1}}+\frac{1}{n_{b}}}
\end{aligned}
$$

Thus, we form a new normalized decision matrix, Equation (18):

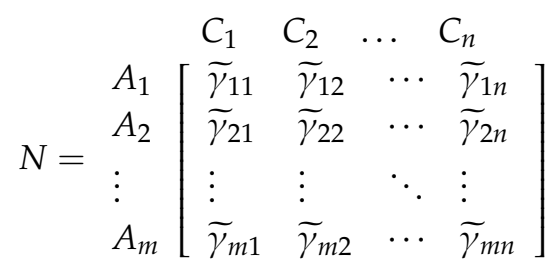

where $\widetilde{\gamma}_{i j} \in[0,1]$ represent the normalized elements of the matrix $N$.

Step 5: Calculation of fuzzy criterion functions of alternatives $\widetilde{Q}\left(A_{i}\right)$ and ranking the alternatives. By applying Equation (19), the criterion functions of alternatives $\widetilde{Q}\left(A_{i}\right)$ are calculated and the ranking of alternatives is performed.

$$
\widetilde{Q}\left(A_{i}\right)=\sum_{j=1}^{n} w_{j} \widetilde{\gamma}_{i j}
$$

From the considered set of alternatives, the alternative that has a higher value of fuzzy criterion function $\widetilde{Q}\left(A_{i}\right)$ is selected. In order to simplify the ranking of criterion functions $\widetilde{Q}\left(A_{i}\right)$, it is proposed defuzzification using the equation $Q\left(A_{i}\right)=\left(Q\left(A_{i}\right)^{l}+4 \cdot Q\left(A_{i}\right)^{s}+Q\left(A_{i}\right)^{u}\right) / 6$.

\section{Application of Fuzzy LBWA-MACBETH-RAFSI Multi-Criteria Model}

This section presents the application of a proposed multi-criteria methodology for the selection of strategic guidelines for the reorganization of the healthcare system under the conditions of the COVID-19 pandemic in the Republic of Serbia.

For the evaluation of strategic guidelines, five criteria were defined: $C 1-$ Assessment of which strategic guideline provides the best results in circumstances considered; C2-Assessment of the quality of services provided for patients treated in an infectious disease ward of a clinical center; C3-Assessment of the quality of services provided in non-infectious disease units of a clinical 
center; $C 4-$ Assessment of the effectiveness of preventing the spread of infection in the considered region/country; C5-Assessment of the ratio of the cost for implementing the strategic guideline to the quality of performance of the healthcare center within the strategic guideline. The criteria were defined on the basis of a questionnaire survey of 30 experts who participated in the work of the Republic Crisis Response Team. Most of the surveyed experts are medical professionals. The values of the weights of the criteria are directly dependent on infection capacity and pandemic spread risk $(\delta)$. Depending on infection capacity and pandemic spread risk $(\delta)$, the values of weight coefficients of criteria are defined and a strategic guideline for the reorganization of the healthcare system is selected. Based on statistical reports and experts' estimation, the Republic Crisis Response Team have defined the values $p=1.8$ and $\theta=3$, on the basis of which we obtain $\delta=5.4$. Since the value $\delta=5.4$ is in the interval $5.0<\delta \leq 7.0$, this implies that there is a high infection capacity and pandemic spread risk (Table 2). Based on the defined value $\delta$, experts of the crisis response team were surveyed and the values of weight coefficients were defined using the LBWA model.

\section{(a) Application of LBWA model}

The following section presents the procedure for defining weight coefficients applying the LBWA model:

Step 1: Determining the most significant criterion from the set of criteria $S=\left\{C_{1}, C_{2}, \ldots, C_{5}\right\}$. A total of 30 experts from three crisis response teams participated in the research. Since the experts were grouped within the crisis response teams, group decisions were submitted by each crisis response team separately. Thus, in this study, each crisis response team is viewed as a separate expert preference. As the most significant/influential criterion, all three expert groups (EG1,EG2 and EG3) defined the criterion $C_{1}$.

Step 2. Grouping criteria within significance levels. In accordance with expert preferences, the criteria are grouped into the following levels:

$$
\begin{aligned}
& E G 1 \text { and } E G 3: \\
& L_{1}=\left\{C_{1}, C_{2}, C_{3}\right\}, \\
& L_{2}=\left\{C_{4}\right\}, \\
& L_{3}=\left\{C_{5}\right\} . \\
& E G 2: \\
& L_{1}=\left\{C_{1}, C_{2}, C_{3}\right\}, \\
& L_{2}=\left\{C_{4}\right\}, \\
& L_{3}=\varnothing \\
& L_{4}=\left\{C_{5}\right\} .
\end{aligned}
$$

Step 3. Based on Equation (2), the maximum value of scale $(\lambda)$ for the evaluation of criteria within the significance levels is defined.

$$
\begin{aligned}
& \text { EG1 and } E G 3 \text { : } \\
& \left.\begin{array}{l}
L_{1}=\left\{C_{1}, C_{2}, C_{3}\right\}, \\
L_{2}=\left\{C_{4}\right\}, \\
L_{3}=\left\{C_{5}\right\} .
\end{array}\right\} \Rightarrow \lambda=\max \left\{\left|L_{1}\right|,\left|L_{2}\right|,\left|L_{3}\right|\right\}=3 \\
& \text { EG2 : } \\
& L_{1}=\left\{C_{1}, C_{2}, C_{3}\right\} \text {, } \\
& L_{2}=\left\{C_{4}\right\} \text {, } \\
& L_{3}=\varnothing \\
& L_{4}=\left\{C_{5}\right\} \text {. } \\
& \} \lambda=\max \left\{\left|L_{1}\right|,\left|L_{2}\right|,\left|L_{3}\right|,\left|L_{4}\right|\right\}=3
\end{aligned}
$$


The scale for the evaluation of criteria is defined for each expert group separately. The maximum number of criteria within the significance levels is the same for all three expert groups. Therefore, the same scale for evaluation of criteria $I \in[0,3]$ was defined. Comparisons of the criteria by expert groups EG1, EG2 and EG3 are shown in Table 4.

Table 4. Comparisons of the criteria within significance levels by EG1, EG2 and EG3.

\begin{tabular}{cccc}
\hline Criteria & EG1 & EG2 & EG3 \\
\hline C1 & Level $1 ; I=0.0$ & Level $1 ; I=0.0$ & Level $1 ; I=0.0$ \\
C2 & Level 1; I=1.0 & Level $1 ; I=1.2$ & Level $1 ; I=1.5$ \\
C3 & Level 1; $I=1.5$ & Level $1 ; I=1.4$ & Level $1 ; I=1.7$ \\
C4 & Level 2; $I=0.5$ & Level $2 ; I=1.0$ & Level $2 ; I=0.8$ \\
C5 & Level 3; $I=0.0$ & Level $4 ; I=0.0$ & Level 3; $I=0.3$ \\
\hline
\end{tabular}

Step 4. Based on the value $\lambda$ and conditions that the elasticity coefficient is $\Delta \lambda>\lambda$, $\lambda=\max \left\{\left|L_{1}\right|,\left|L_{2}\right|, \ldots,\left|L_{l}\right|\right\}$, in this research, the value $\Delta \lambda=4$ is taken as the elasticity coefficient.

Step 5. Defining the influence function of the criteria. By applying Equation (4), the influence functions of the criteria for each expert group are calculated:

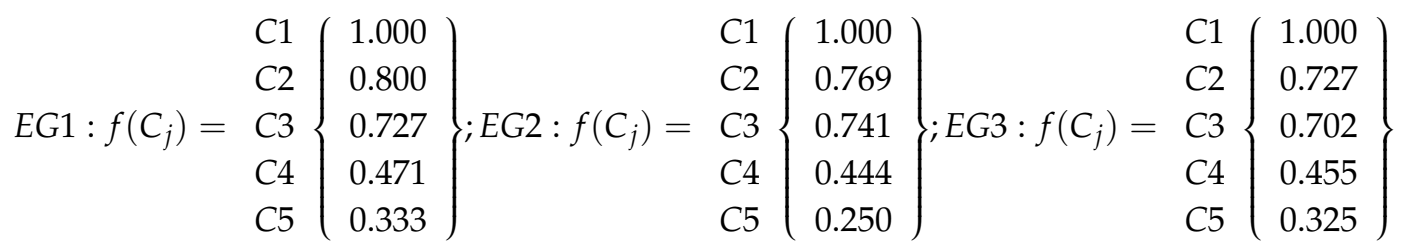

Thus, for the criterion $C 2$ from EG1, by applying Equation (4), we obtain the value $f\left(C_{2}\right)=4 /(1 \cdot 4+1)=0.800$. In a similar way, we obtain the remaining values of the influence function of the criteria.

Step 6. Calculation of optimal values of the weight coefficients of the criteria. By applying Equation (5), we obtain the weight coefficient of the best criterion (C1).

$$
\begin{gathered}
E G 1: w_{1}=\frac{1}{1+0.800+0.727+0.471+0.333}=0.300 \\
E G 2: w_{1}=\frac{1}{1+769+0.741+0.444+0.250}=0.312 \\
E G 3: w_{1}=\frac{1}{1+0.727+0.702+0.455+0.325}=0.312
\end{gathered}
$$

The values of the weight coefficients of the remaining criteria are obtained by applying Equation (6).

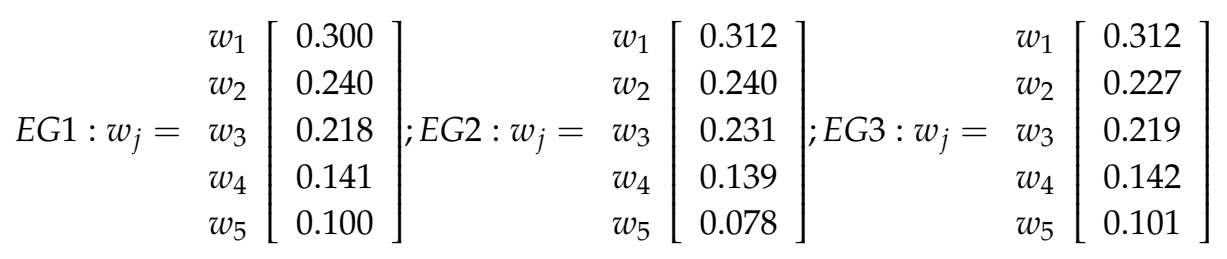

By arithmetic averaging, we obtain the final values of weight coefficients $w_{j}^{L B W A}=$ $(0.308,0.236,0.223,0.141,0.093)^{T}$.

(b) Application of MACBETH model:

The following section presents the procedure for defining weight coefficients applying the MACBETH model. 
In the first step, the criteria were discussed with the decision makers in the expert groups and the group decision can be seen in Table 5 .

Table 5. Pairwise comparison matrix of expert groups.

\begin{tabular}{|c|c|c|c|c|}
\hline Crit. & $C 2$ & $C 3$ & $C 4$ & $C 5$ \\
\hline $\mathrm{C} 1$ & moderate & strong & moderate & moderate \\
\hline $\mathrm{C} 2$ & & weak & weak & weak \\
\hline C3 & & & moderate & moderate \\
\hline$C 4$ & & & & weak \\
\hline
\end{tabular}

According to the decision makers' evaluations, the linear programming model (7) is constructed as follows:

$$
\begin{aligned}
& z_{\min }=\phi\left(o_{1}\right) \\
& \phi\left(o_{1}\right) \geq \phi\left(o_{2}\right)+3 ; \phi\left(o_{1}\right) \geq \phi\left(o_{3}\right)+5 ; \ldots ; \\
& \phi\left(o_{3}\right) \geq \phi\left(o_{5}\right)+3 ; \phi\left(o_{4}\right) \geq \phi\left(o_{5}\right)+2 ; \\
& \phi\left(o_{1}\right)-\phi\left(o_{2}\right) \geq \phi\left(o_{4}\right)-\phi\left(o_{5}\right)+1 ; \\
& \phi\left(o_{1}\right)-\phi\left(o_{3}\right) \geq \phi\left(o_{4}\right)-\phi\left(o_{5}\right)+3 ; \\
& \ldots \\
& \phi\left(o_{3}\right)-\phi\left(o_{4}\right) \geq \phi\left(o_{4}\right)-\phi\left(o_{5}\right)+1 ; \\
& \phi\left(o_{3}\right)-\phi\left(o_{5}\right) \geq \phi\left(o_{4}\right)-\phi\left(o_{5}\right)+1 ; \\
& \phi\left(o_{5}\right)=1 \\
& \forall \phi\left(o_{j}\right) \geq 0 ; j=1,2, \ldots, 5 ;
\end{aligned}
$$

By solving the linear model, we get the criterion values $\phi\left(o_{j}\right)=(11,8,6,3,1)$. By applying Equation (8), the values $\phi\left(o_{j}\right)$ are normalized, and we obtain the final values of the weighting coefficients according to the MACBETH methodology $w_{j}^{M A C B E T H}=(0.379,0.276,0.207,0.103,0.034)$.

Finally, based on the obtained values of the weights of the criteria according to the LBWA and MACBETH methodology, using Equation (9) we obtain the combined values of the weighting coefficients; see Table 6.

Table 6. Combined values of weight coefficients.

\begin{tabular}{cccc}
\hline Criteria & LBWA & MACBETH & Final Weights \\
\hline C1 & 0.308 & 0.379 & 0.344 \\
C2 & 0.236 & 0.276 & 0.256 \\
C3 & 0.223 & 0.207 & 0.215 \\
C4 & 0.141 & 0.103 & 0.122 \\
C5 & 0.093 & 0.034 & 0.064 \\
\hline
\end{tabular}

To obtain aggregated values of weighting coefficients, a value $\xi=0.5$ was used, whereby both methodologies equally participate in defining the final weights of the criteria.

\section{(C) Application of fuzzy RAFSI model:}

After defining the weight coefficients of the criteria, the experts from the crisis response teams evaluated the strategic guidelines using the fuzzy RAFSI model. The following section presents the application of the fuzzy RAFSI model for the evaluation of four strategic guidelines for the reorganization of the healthcare system for the value $\delta=5.4$. The experts evaluated the strategic guidelines $A_{i}(i=1,2, \ldots, 4)$ in relation to five criteria $C_{j}(j=1,2, \ldots, 5)$ defined in the previous section of the paper. All criteria $C_{j}(j=1,2, \ldots, 5)$ belong to the group of max criteria. To evaluate the strategic guidelines (alternatives), the experts used a fuzzy linguistic scale shown in Table 7. 
Table 7. Fuzzy linguistic scale for evaluating alternatives $[29,30]$.

\begin{tabular}{cc}
\hline Linguistic Terms & Membership Function \\
\hline Very poor (VP) & $(1,1,1)$ \\
Poor (P) & $(1,2,3)$ \\
Medium poor (MP) & $(2,3,4)$ \\
Medium (M) & $(3,4,5)$ \\
Medium high (MH) & $(4,5,6)$ \\
High (H) & $(5,6,7)$ \\
Very high (VH) & $(6,7,8)$ \\
Extremely high (EH) & $(7,8,9)$ \\
Absolutely high (AH) & $(8,9,9)$ \\
\hline
\end{tabular}

After the evaluation of the strategic guidelines, expert correspondence matrices are obtained, which are presented in the following section:

\begin{tabular}{|c|c|c|c|c|c|c|}
\hline \multirow{5}{*}{$X^{(e)}=$} & & $C_{1}$ & $C_{2}$ & $C_{3}$ & $C_{4}$ & $C_{5}$ \\
\hline & $A_{1}$ & $\mathrm{H} ; \mathrm{MP} ; \mathrm{H}$ & $\mathrm{M} ; \mathrm{H} ; \mathrm{H}$ & $\mathrm{MH} ; \mathrm{MH} ; \mathrm{H}$ & $\mathrm{H} ; \mathrm{H} ; \mathrm{VH}$ & $\mathrm{MH} ; \mathrm{VH} ; \mathrm{VH}$ \\
\hline & $A_{2}$ & $\mathrm{H} ; \mathrm{H} ; \mathrm{M}$ & $\mathrm{M} ; \mathrm{M} ; \mathrm{H}$ & $\mathrm{H} ; \mathrm{MH} ; \mathrm{M}$ & $\mathrm{MH} ; \mathrm{H} ; \mathrm{H}$ & $\mathrm{VH} ; \mathrm{H} ; \mathrm{VH}$ \\
\hline & $A_{3}$ & $\mathrm{VH} ; \mathrm{H} ; \mathrm{VH}$ & VH; EH; EH & $\mathrm{H} ; \mathrm{EH} ; \mathrm{VH}$ & $\mathrm{H} ; \mathrm{VH} ; \mathrm{MH}$ & MH; MH; H \\
\hline & $A_{4}$ & $\mathrm{VH} ; \mathrm{VH} ; \mathrm{H}$ & $\mathrm{H} ; \mathrm{VH} ; \mathrm{VH}$ & $\mathrm{MH} ; \mathrm{VH} ; \mathrm{VH}$ & $\mathrm{VH} ; \mathrm{H} ; \mathrm{VH}$ & $\mathrm{H} ; \mathrm{H} ; \mathrm{MH}$ \\
\hline
\end{tabular}

By applying Equation (11), we obtain an averaged initial decision matrix:

$$
\begin{aligned}
& C_{1} \\
& \mathrm{C}_{2} \\
& \mathrm{C}_{3} \\
& \mathrm{C}_{4} \\
& C_{5} \\
& X=\begin{array}{c}
A_{1} \\
A_{2} \\
A_{3} \\
A_{4}
\end{array}\left[\begin{array}{llllll}
(3.87,4.90,5.92) & (4.28,5.29,6.30) & (4.32,5.32,6.32) & (5.32,6.32,7.33) & (5.29,6.30,7.30) \\
(5.66,6.66,7.66) & (3.61,4.62,5.63) & (3.96,4.97,5.97) & (4.65,5.66,6.66) & (5.66,6.66,7.66) \\
(5.66,7.66) & (5.66,6.66,7.66) & (5.97,6.98,7.98) & (4.97,5.97,6.98) & (4.32,5.32,6.32) \\
(5.29,6.30,7.30) & (5.66,6.66,7.66) & (4.65,5.66,6.66)
\end{array}\right]
\end{aligned}
$$

Step 1: Experts defined an ideal $\widetilde{\xi}_{I_{j}}$ and anti-ideal $\widetilde{\xi}_{N_{j}}$ value for each criterion $C_{j}(i=1,2, \ldots, 5)$. For the considered example, by consensus, the decision makers defined the following ideal and anti-ideal points: $\widetilde{\xi}_{I_{j}}=(10,10.5,11.0)$ and $\widetilde{\xi}_{N_{j}}=(0.5,0.5,1.0)$. Since the same linguistic scale have been used to evaluate all criteria, the same ideal and anti-ideal points, $\widetilde{\xi}_{I_{j}}=(10,10.5,11.0)$ and $\widetilde{\xi}_{N_{j}}=(0.5,0.5,1.0)$, apply to all criteria.

Step 2: Based on the defined ideal and anti-ideal points, criterion intervals $C_{j} \in\left[\widetilde{\xi}_{N_{j}}, \widetilde{\xi}_{I_{j}}\right]$ are formed, where $\widetilde{\xi}_{N_{j}}=(0.5,0.5,1.0)$ and $\widetilde{\xi}_{I_{j}}=(10,10.5,11.0)$. In this research, it is defined that the ideal value of the alternative is nine times higher than the anti-ideal value, i.e., that $n_{1}=1$ and $n_{s}=9$. By applying Equation (13), we define the functions on the basis of which the standardization of criteria is performed, i.e., the transformation of the values of the initial decision matrix into an interval $[1,9]$. Thus, we obtain the following fuzzy functions:

$$
\widetilde{f}_{A_{i}}\left(C_{j}\right)=\frac{9-1}{(10,10.5,11)-(0.5,0.5,1.0)} \widetilde{\xi}_{i j}+\frac{(10,10.5,11) \cdot 1-(0.5,0.5,1.0) \cdot 9}{(10,10.5,11)-(0.5,0.5,1.0)}
$$

i.e.,

$$
\begin{aligned}
& \widetilde{f}_{A_{i}}\left(C_{j}\right)=\left(f_{i j}^{l}\left(C_{j}\right), f_{i j}^{s}\left(C_{j}\right), f_{i j}^{u}\left(C_{j}\right)\right) \\
& =\left\{\begin{array}{c}
f_{A_{i}}^{l}\left(C_{1}\right)=\frac{9-1}{10-1} \xi_{i j}^{l}+\frac{10 \cdot 1-1.9}{10-1}=0.89 \cdot \xi_{i j}^{l}+0.11 \\
f_{A_{i}}^{s}\left(C_{1}\right)=\frac{9-1}{10.0-0.5} \xi_{i j}^{s}+\frac{10 \cdot 1-0.5 \cdot 9}{10.5-0.5}=0.80 \cdot \xi_{i j}^{s}+0.60 \\
f_{A_{i}}^{u}\left(C_{1}\right)=\frac{9-1}{11-0.5} \xi_{i j}^{u}+\frac{11 \cdot 1-0.5 \cdot 9}{11-0.5}=0.76 \cdot \xi_{i j}^{s}+0.62
\end{array}\right.
\end{aligned}
$$

where $\widetilde{\xi}_{i j}=\left(\xi_{i j^{\prime}}^{l}, \xi_{i j^{\prime}}^{s}, \xi_{i j}^{u}\right)$ represent elements of the aggregated initial decision matrix. 
Since all criteria values in the initial decision matrix cover the same value interval, i.e., they are defined using the same linguistic scale, the same function $\widetilde{f}_{A_{i}}\left(C_{j}\right)$ is applied to map all criteria $\left.C_{j} j=1,2, \ldots, 5\right)$.

By applying the function $\widetilde{f}_{A_{i}}\left(C_{j}\right)$, we obtain a standardized decision matrix $\left(T=\left[\widetilde{\varphi}_{i j}\right]_{4 \times 5}\right.$, $i=1,2, \ldots, 4, j=1,2, \ldots, 5)$ in which all elements of the matrix are transformed into an interval $[1,9]$.

\begin{tabular}{|c|c|c|c|c|c|}
\hline & $C 1$ & $C 2$ & C3 & $C 4$ & C5 \\
\hline$A 1$ & $(3.55,4.52,5.13)$ & $(3.91,4.83,5.42)$ & $(3.95,4.86,5.44)$ & $(4.84,5.66,6.20)$ & $(4.81,5.64,6.18)$ \\
\hline$T-A 2$ & $(3.91,4.83,5.42)$ & $(3.31,4.30,4.91)$ & $(3.62,4.57,5.17)$ & $(4.24,5.13,5.69)$ & $(5.13,5.93,6.45)$ \\
\hline$I=A 3$ & $(5.13,5.93,6.45)$ & $(6.02,6.73,7.22)$ & $(5.41,6.18,6.70)$ & $(4.52,5.38,5.93)$ & $(3.95,4.86,5.44)$ \\
\hline$A 4$ & $(5.13,5.93,6.45)$ & $(5.13,5.93,6.45)$ & $(4.81,5.64,6.18)$ & $(5.13,5.93,6.45)$ & $(4.24,5.13,5.69)$ \\
\hline
\end{tabular}

By substituting the values from the matrix $X$, Equation (12), into the function $\widetilde{f}_{A_{i}}\left(C_{j}\right)$, Equation (13), we obtain the remaining values of the elements $\widetilde{\varphi}_{i j}$. Elements at position $A_{i}-C_{1}$ are obtained by applying the function $\widetilde{f}_{A_{i}}\left(C_{j}\right)$ :

$$
\begin{gathered}
\widetilde{f}_{A_{1}}\left(C_{1}\right)=(3.55,4.52,5.13)=\left\{\begin{array}{l}
f_{A_{1}}^{l}\left(C_{1}\right)=0.89 \cdot \xi_{11}^{l}+0.11=0.89 \cdot 3.87+0.11=3.55 \\
f_{A_{1}}^{s}\left(C_{1}\right)=0.80 \cdot \xi_{11}^{s}+0.60=0.80 \cdot 4.90+0.60=4.52 \\
f_{A_{1}}^{u}\left(C_{1}\right)=0.76 \cdot \xi_{11}^{u}+0.62=0.76 \cdot 5.92+0.62=5.13
\end{array}\right. \\
\widetilde{f}_{A_{2}}\left(C_{1}\right)=(3.91,4.83,5.42)=\left\{\begin{array}{l}
f_{A_{2}}^{l}\left(C_{1}\right)=0.89 \cdot 4.28+0.11=3.91 \\
f_{A_{2}}^{s}\left(C_{1}\right)=0.80 \cdot 5.29+0.60=4.83 \\
f_{A_{2}}^{u}\left(C_{1}\right)=0.76 \cdot 6.30+0.62=5.42
\end{array}\right. \\
\widetilde{f}_{A_{3}}\left(C_{1}\right)=(5.13,5.93,6.45)=\left\{\begin{array}{l}
f_{A_{3}}^{l}\left(C_{1}\right)=0.89 \cdot 5.66+0.11=5.13 \\
f_{A_{3}}^{s}\left(C_{1}\right)=0.80 \cdot 6.66+0.60=5.93 \\
f_{A_{3}}^{u}\left(C_{1}\right)=0.76 \cdot 7.66+0.62=6.45
\end{array}\right. \\
\widetilde{f}_{A_{4}}\left(C_{1}\right)=(5.13,5.93,6.45)=\left\{\begin{array}{l}
f_{A_{3}}^{l}\left(C_{1}\right)=0.89 \cdot 5.66+0.11=5.13 \\
f_{A_{3}}^{s}\left(C_{1}\right)=0.80 \cdot 6.66+0.60=5.93 \\
f_{A_{3}}^{u}\left(C_{1}\right)=0.76 \cdot 7.66+0.62=6.45
\end{array}\right.
\end{gathered}
$$

Step 3: We calculate the arithmetic mean and the harmonic mean of the minimum and maximum elements, $n_{1}=1$ and $n_{b}=9$ :

$$
\begin{gathered}
A=\left(n_{1}+n_{b}\right) / 2=(1+9) / 2=5 \\
H=\frac{2}{\frac{1}{n_{1}}+\frac{1}{n_{b}}}=\frac{2}{\frac{1}{9}+\frac{1}{1}}=1.80
\end{gathered}
$$

So, for $n_{1}=1$ and $n_{b}=9$, we obtain that the arithmetic mean is $A=5.5$, while the harmonic mean is $H=1.80$.

Step 4: By applying Equation (15), the elements of the matrix $T$ are normalized and transformed depending on whether they belong to the min or max type of criteria. Since, in our example, all the criteria are of the max type, only the arithmetic mean will be used to normalize the elements of the $T$ matrix. So, we obtain a new matrix $N=\left[\widetilde{\gamma}_{i j}\right]_{4 \times 5}(i=1,2, \ldots, 4, j=1,2, \ldots, 5)$, Equation (18).

$$
\begin{aligned}
& \text { C1 }
\end{aligned}
$$

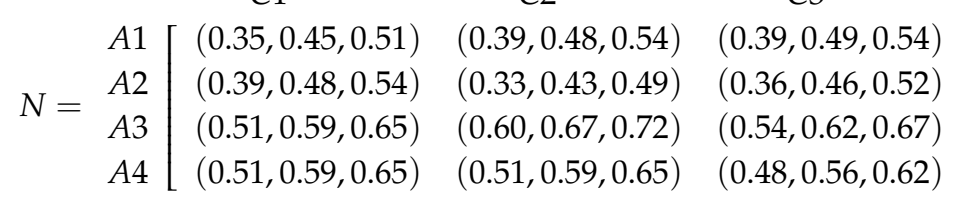

$$
\begin{aligned}
& \text { C4 } \\
& \begin{array}{lll}
(0.48,0.57,0.62) & (0.48,0.56,0.62)
\end{array} \\
& \begin{array}{ll}
(0.42,0.51,0.57) \quad(0.51,0.59,0.65) \\
\hline
\end{array} \\
& (0.45,0.54,0.59) \quad(0.39,0.49,0.54) \\
& (0.51,0.59,0.65) \\
& (0.42,0.51,0.57)
\end{aligned}
$$


Thus, for example, for criterion $C 1$, at position $A 1-C 1$, we obtain that $\widetilde{\gamma}_{11}=\frac{(3.55,4.52,5.13)}{2 \cdot 5}=$ $\left(\frac{3.55}{10}, \frac{4.52}{10}, \frac{5.13}{10}\right)=(0.355,0.452,0.513)$.

Step 5: By applying Equation (19), the criterion functions of alternatives $\widetilde{Q}\left(A_{i}\right)$ are calculated. The alternative is ranked on the basis of value $\widetilde{Q}\left(A_{i}\right)$, so it is more desirable that the alternative has a higher value $\widetilde{Q}\left(A_{i}\right)$.

The ranking of alternatives is shown in Table 8.

Table 8. Criterion functions and final ranking of alternatives.

\begin{tabular}{cccc}
\hline Alt. & $\widetilde{Q}\left(A_{i}\right)$ & Crisp $Q\left(A_{i}\right)$ & Rank \\
\hline$A 1$ & $(0.397,0.489,0.547)$ & 0.4829 & 3 \\
$A 2$ & $(0.382,0.475,0.534)$ & 0.4690 & 4 \\
$A 3$ & $(0.527,0.605,0.658)$ & 0.6011 & 1 \\
$A 4$ & $(0.501,0.582,0.635)$ & 0.5771 & 2 \\
\hline
\end{tabular}

Based on the obtained results, we can single out the strategic guideline A3 as the dominant solution.

\section{Validation of the Results}

The validation of the results was carried out through two sections. The first section considers the analysis of the influence of changing parameters $p$ and $q$ in the Bonferroni integration operator. The second section presents the validation of the results through comparison with other multi-criteria techniques.

\subsection{Influence of Changing Parameters $p$ and $q$ on the Results of Ranking}

The Bonferroni integration operator was used to integrate expert preferences into an aggregated initial decision matrix. During the formation of the aggregated initial decision matrix on the basis of which the initial rank was obtained (Table 8), the values of the parameters $p=q=1$ were adopted. Considering the mathematical equation of the Bonferroni integration operator, it is clear that different values of parameters $p$ and $q$ lead to the transformation of the mathematical Equation $(11)[31,32]$. Having in mind the stated facts, it is necessary to examine the influence of changing parameters $p$ and $q$ on changing the values in the aggregated decision matrix. Additionally, changes in values in the aggregate decision matrix indirectly lead to changes in the values of criterion functions and potential changes in the ranks of strategic guidelines. Therefore, in the following section, the influence of changing parameters $p$ and $q$ on changing the values of the criterion functions of strategic guidelines is analyzed.

In order to validate the results, the change in parameters $p$ and $q$ was simulated and analyzed in the interval $[1,100]$ through 300 scenarios. In the first 200 scenarios, the impact of changing only one parameter of the parameters in the interval $[1,100]$ was analyzed, while the other parameter had the value one. Thus, through the first 100 scenarios, the change in the parameter value $1 \leq p \leq 100$ was simulated, while in the next 100 scenarios, the change in the parameter $1 \leq q \leq 100$ was simulated; see Figure 3a. In the last 100 scenarios, the change in both parameters in the interval $1 \leq p, q \leq 100$ was simulated; see Figure $3 b$.

By analyzing the results from Figure 3, we notice that for changes in the values of parameters $1 \leq p, q \leq 100$ through all 300 scenarios, there is no change in the ranks of strategic guidelines and that the initial rank $A 4>A 3>A 2>A 1$ is confirmed. We notice that the change in the values of the parameters in the interval $1 \leq p, q \leq 100$ leads to a change in the values of criterion functions. However, changes in the values of the criterion functions of the strategic guidelines are not large enough to cause changes in the initial rank. In addition, the results presented show that the initial ordering is credible and robust despite drastic changes in input parameters. It is necessary to emphasize that such an analysis is an indispensable step in multi-criteria models in which integration functions are 
used for the aggregation of input parameters. The integration functions have free parameters whose values are arbitrarily determined by decision makers. This is confirmed by numerous studies that have shown that changes in the values of integration function parameters can often lead to changes in initial ranks [33-35].

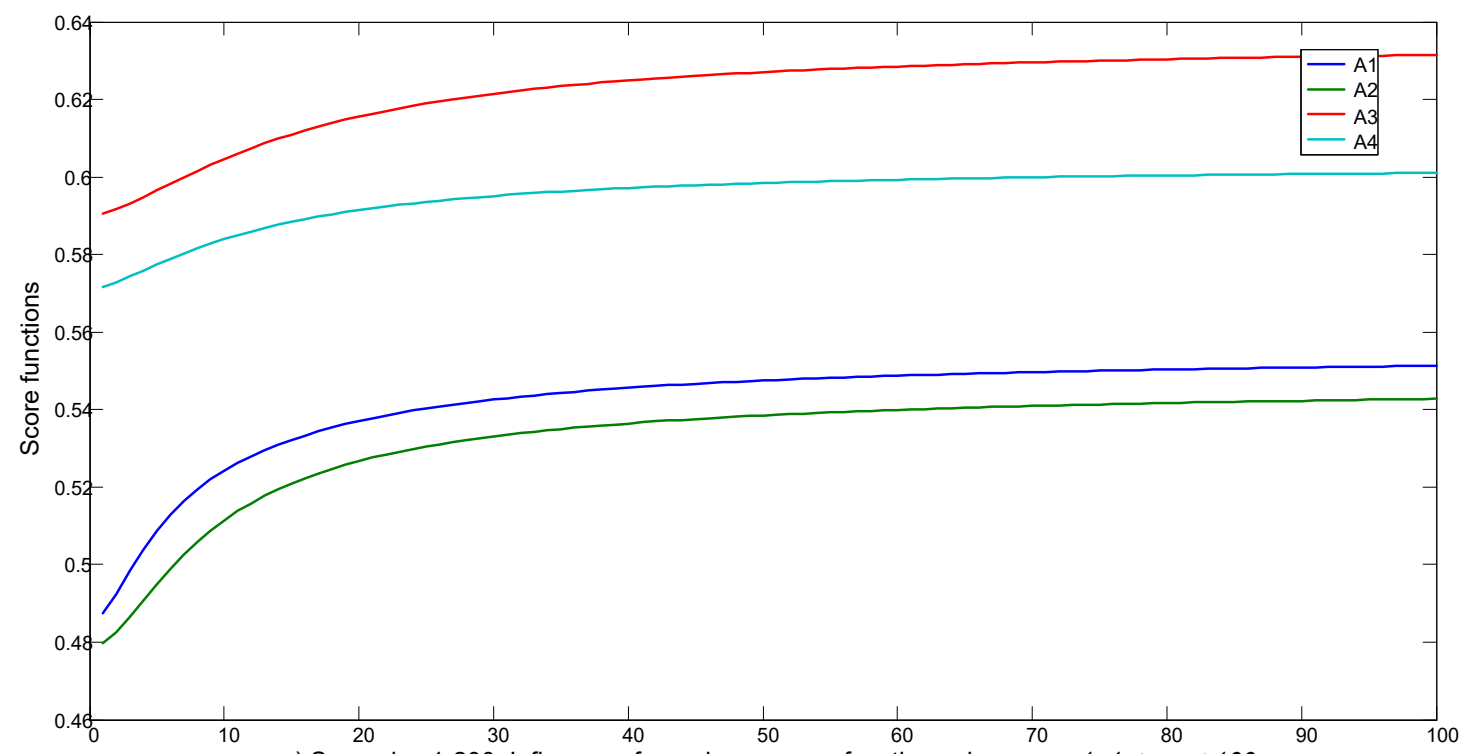

a) Scenarios 1-200: Influence of $p$ and $q$ on score function value $-p, q=1,1 \leq p, q \leq 100$

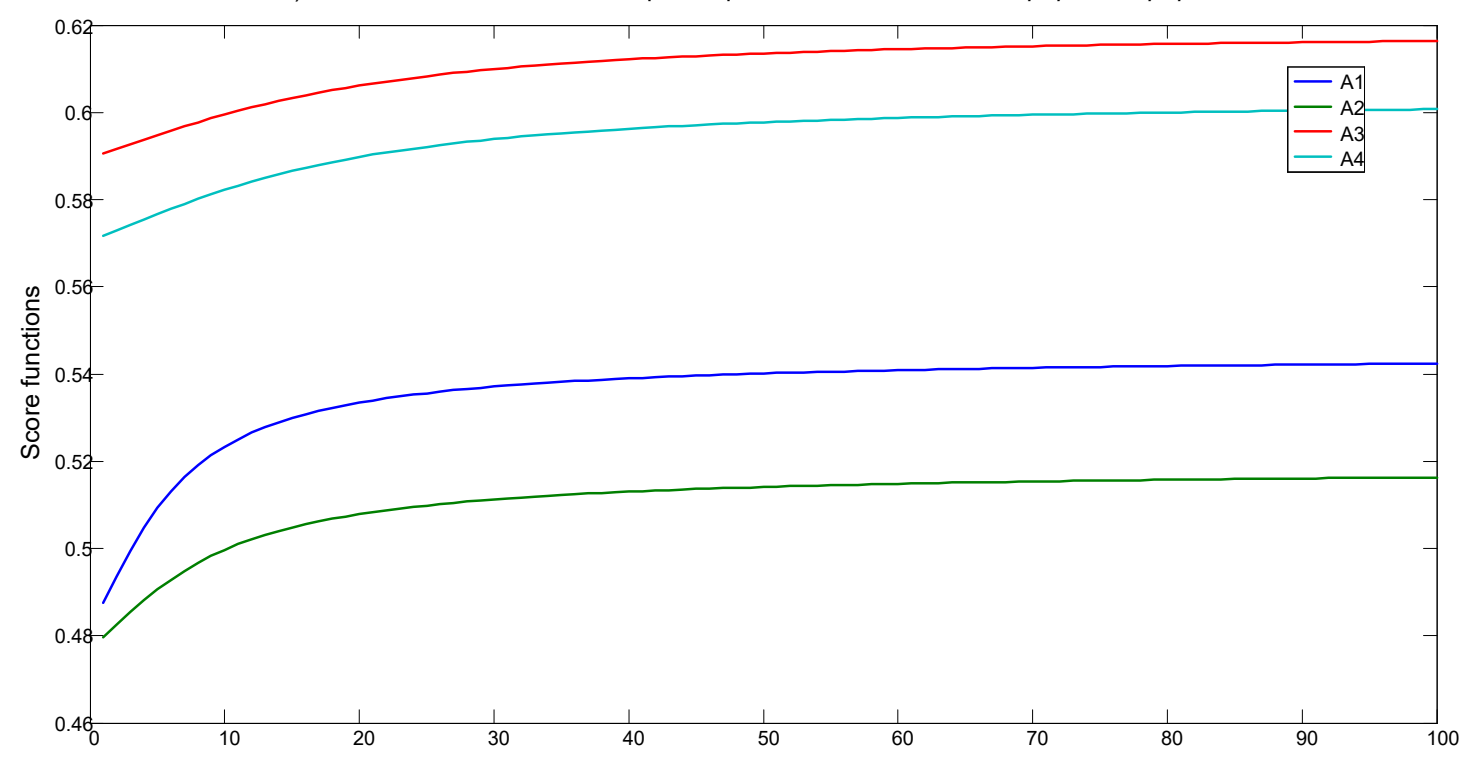

b) Scenarios 200-300: Influence of $p$ and $q$ on score function value $-1 \leq p, q \leq 100 ; p=q$

Figure 3. Influence of parameters $p$ and $q$ on changing the utility function of strategic guidelines.

\subsection{Computation of Stability of Ranking Based on Different Ranking Methodologies}

In order to validate the initial rank, four more multi-criteria techniques were applied under the same conditions (with the same input values) as for the RAFSI method: Compressed Proportional Assessment (COPRAS) [36], Multi-Attributive Ideal-Real Comparative Analysis (MAIRCA) [37], Multi-Attributive Border Approximation area Comparison (MABAC) [38] and Measurement of Alternatives and Ranking According to Compromise Solution (MARCOS) [39]. A comparative overview of the ranks obtained by applying the above multi-criteria techniques is shown in Figure 4.

Based on the comparison of results shown in Figure 4, the order of ranking of the strategic guidelines according to the LBWA-MACBETH-RAFSI model is similar to the order proposed by the multi-criteria techniques developed in [36-39]. The presented analysis proves the validity of 
the methodology proposed in this paper and that the proposed selection of strategic guideline A3 is credible.

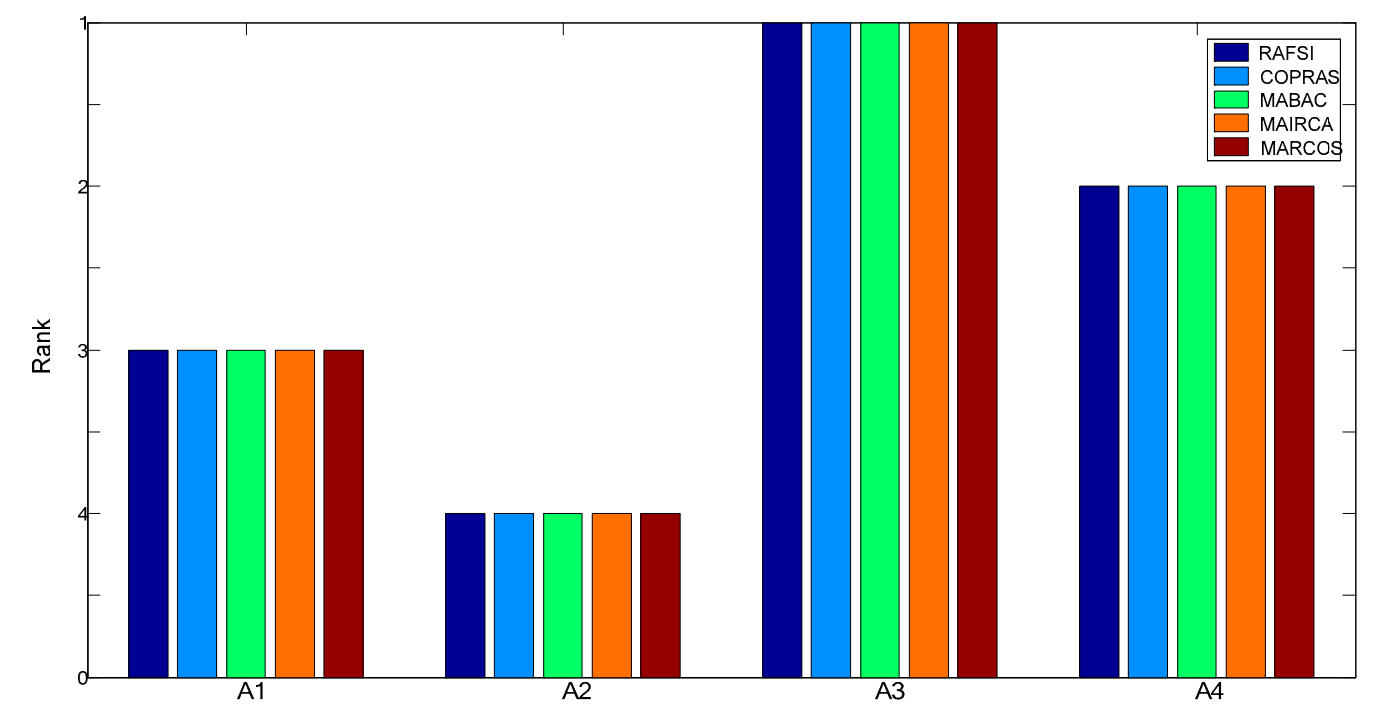

Figure 4. The comparison of different MCDM methodologies.

\section{Conclusions}

The adaptability of healthcare systems is a key segment that affects the efficiency of the healthcare system of each country. This fact is particularly evident in crisis situations, such as the COVID-19 pandemic, which has affected the whole world. Therefore, it is necessary to efficiently manage the organization of healthcare systems in order to provide adequate medical care to patients and reduce dangers for the population caused by the COVID-19 pandemic to a minimum. This paper presents a multi-criteria model that proposes strategic guidelines for possible adaptation of healthcare system depending on infection capacity and pandemic spread risk. Four strategic guidelines have been defined and five criteria for the evaluation of the strategic guidelines have been determined. The paper proposes a scale on the basis of which infection capacity and pandemic spread risk are defined. Based on the estimation of infection capacity and pandemic spread risk by the crisis response team, the weight coefficients of criteria are defined and a strategic guideline is selected for the possible reorganization of a healthcare system of a region within a country or a whole country. The LBWA and MACBETH methods were proposed to define the values of the weight coefficients of the criteria, while the novel fuzzy RAFSI method was proposed for the evaluation of strategic guidelines and for the proposal of the possible organization of healthcare systems.

The proposed multi-criteria methodology was tested for values of high infection capacity and pandemic spread risk $(1.5<\delta \leq 2.0)$. The application of the fuzzy LBWA-MACBETH-RAFSI methodology was demonstrated and the results were validated. Based on the validation of the results, strategic guideline S3 was proposed as the best solution for the reorganization of the healthcare system under the conditions of a high infection capacity and pandemic spread risk. The testing was performed using the example of the healthcare system in the Republic of Serbia.

The proposed methodology has several contributions that can be observed from two perspectives. The first standpoint refers to the contribution of the proposed methodology within the healthcare filed, where we can highlight the following advantages: (1) an original methodology for reorganization and adjustment of healthcare systems in emergency situations caused by the COVID-19 pandemic has been proposed; (2) a methodology for determining infection capacity and pandemic spread risk has been defined, which is a prerequisite for the application of a multi-criteria methodology; (3) strategic guidelines and criteria for the evaluation of the guidelines were defined on the basis of real research conducted during the COVID-19 pandemic and in which medical experts and other members of the crisis response team in Serbia participated; (4) the proposed methodology provides a new framework 
for managing the organization of healthcare systems under the dynamic conditions of a pandemic. The model enables national policy makers to establish strategic guidelines for adapting healthcare systems to the capacity of the COVID-19 pandemic; (5) the universality of the proposed multi-criteria methodology allows the application of the proposed methodology for the reorganization of healthcare systems of other countries with minor pre-adjustments of strategic guidelines and criteria depending on the specific characteristics of the country or region in which it is applied.

The second standpoint refers to the contributions of the proposed methodology within the MCDM area, where we can highlight the following contributions: (1) within the proposed multi-criteria methodology, an original extension of the RAFSI method in a fuzzy environment was performed; (2) the fuzzy LBWA-MACBETH-RAFSI model has a flexibility and universality that allow it to be effectively applied to various areas where the application of a rational and robust methodology is required; (3) the hybrid fuzzy LBWA-MACBETH-RAFSI model contributes to the improvement of the literature that considers the theoretical and practical application of multi-criteria techniques; (4) the proposed multi-criteria methodology allows the evaluation of alternatives despite inaccuracies and a lack of quantitative information in a decision-making process and (5) the methodology for the evaluation of healthcare systems in emergency situations is improved through a new approach to treating inaccuracies since the application of this or a similar approach to the healthcare field was not observed in our analysis of the literature.

There are certain limitations that should be considered when interpreting the results of this study. The effectiveness of the proposed fuzzy MCDM tool is evident, but acceptance by other users and decision makers can be a limiting factor. Most users and decision makers readily accept mathematically simple and easy-to-understand decision-making tools. However, tools that require the processing of group information, while respecting the uncertainties and inaccuracies that exist in group preferences, are complex in nature. Therefore, the model used in this paper does not fall into the mathematically simple category of decision-making tools. A particular limitation of this model is the complex mathematical formulations for the fusion of expert preferences into a single group decision. However, integrating the LBWA-MACBETH-RAFSI model into a decision-making system will make it more acceptable for use by heath care management who have to deal with a high degree of uncertainty and inaccuracy during decision making. The proposal of a user-oriented decision support system was made during the preparation of this study and is based on the implementation of Visual Basic and Matlab software packages.

Other limitations of our study relate to the determination of weighting criteria that significantly depend on the subjective characteristics of the available experts participating in the study. Another limitation is the small size of the sample examined and the possible impact of survey formatting on study results. Therefore, it is recommended that further research be organized on a larger sample of respondents familiar with the context of the decision and consider the possibility of increasing the number of criteria that would be grouped into clusters.

The proposed LBWA-MACBETH-RAFSI model is a tool that can be successfully integrated with other MCDM techniques. The development of hybrid multi-criteria models for group decision making, which would be based on the integration of LBWA-MACBETH-RAFSI model and other MCDM tools, is one of the future directions for its application. Another logical step for the future improvement of the LBWA-MACBETH-RAFSI model is its application in other uncertain theories, such as rough, grey, neutrosophic, etc. Numerous linguistic approaches have been developed in recent years, such as extensions of linguistic variables in a neutrosophic environment and an unbalanced linguistic approach. These approaches have attracted a lot of attention in the field of decision making, through the possibility of applying linguistic variables in a decision-making process. Linking these linguistic approaches to the LBWA-MACBETH-RAFSI model and researching the possibility of linguistic modeling of preferences are interesting and promising topics for future research. 
Author Contributions: Conceptualization, methodology, validation, writing —original draft preparation, D.P., M.Ž. and D.M.; review and editing, D.D., S.V.J. and P.B. All authors have read and agreed to the published version of the manuscript.

Funding: We acknowledge support by the German Research Foundation and the Open Access Publication Fund of TU Berlin.

Acknowledgments: The authors wish to acknowledge the support received from German Research Foundation and the TU Berlin.

Conflicts of Interest: The authors declare no conflict of interest.

\section{References}

1. Stankovic, S.M. Banje Srbije; Zavod za Udžbenike: Beograd, Serbia, 2010.

2. Zizovic, M.; Pamucar, D. New model for determining criteria weights: Level Based Weight Assessment (LBWA) model. Decis. Mak. Appl. Manag. Eng. 2019, 2, 126-137. [CrossRef]

3. Bana, E.; Costa, C.A.; Vansnick, J.C. MACBETH-An Interactive Path Towards The Construction of Cardinal Value Functions. Int. Trans. Oper. Res. 1994, 1, 489-500. [CrossRef]

4. Zizovic, M.; Pamucar, D.; Albijanic, M.; Chatterjee, P.; Pribicevic, I. Eliminating Rank Reversal Problem Using a New Multi-Attribute Model-The RAFSI Method. Mathematics 2020, 8, 1015. [CrossRef]

5. Nekoie-Moghadam, M.; Kurland, L.; Moosazadeh, M.; Ingrassia, P.L.; Della Corte, F.; Djalali, A. Tools and checklists used for the evaluation of hospital disaster preparedness: A systematic review. Disaster Med. Public Health Prep. 2016, 10, 781-788. [CrossRef] [PubMed]

6. Fallah-Aliabadi, S.; Ostadtaghizadeh, A.; Ardalan, A.; Fatemi, F.; Khazai, B.; Mirjalili, M.R. Towards developing a model for the evaluation of hospital disaster resilience: A systematic review. BMC Health Serv. Res. 2020, 20, 64. [CrossRef]

7. Verheul, M.L.; Dückers, M.L. Defining and operationalizing disaster preparedness in hospitals: A systematic literature review. Prehosp. Disaster Med. 2020, 35, 61-68. [CrossRef]

8. Alruwaili, A.; Islam, S.; Usher, K. Disaster Preparedness in Hospitals in the Middle East: An Integrative Literature Review. Disaster Med. Public Health Prep. 2019, 13, 806-816. [CrossRef]

9. Tabatabaei, S.A.N.; Abbasi, S. Risk assessment in social security hospitals of Isfahan Province in case of disasters based on the hospital safety index. Int. J. Health Syst. Disaster Manag. 2016, 4, 82.

10. Samsuddin, N.M.; Takim, R.; Nawawi, A.H.; Alwee, S.N.A.S. Disaster preparedness attributes and hospital's resilience in Malaysia. Procedia Eng. 2018, 212, 371-378. [CrossRef]

11. Marzaleh, M.A.; Rezaee, R.; Rezaianzadeh, A.; Rakhshan, M.; Hadadi, G.; Peyravi, M. Emergency Department Preparedness of Hospitals for Radiation, Nuclear Accidents, and Nuclear Terrorism: A Qualitative Study in Iran. Iran. Red Crescent Med. J. 2019, 21. [CrossRef]

12. Shabanikiya, H.; Jafari, M.; Gorgi, H.A.; Seyedin, H.; Rahimi, A. Developing a practical toolkit for evaluating hospital preparedness for surge capacity in disasters. Int. J. Disaster Risk Reduct. 2019, 34, 423-428. [CrossRef]

13. Mulyasari, F.; Inoue, S.; Prashar, S.; Isayama, K.; Basu, M.; Srivastava, N.; Shaw, R. Disaster preparedness: Looking through the lens of hospitals in Japan. Int. J. Disaster Risk Sci. 2013, 4, 89-100. [CrossRef]

14. Hosseini, S.M.; Bahadori, M.; Raadabadi, M.; Ravangard, R. Ranking hospitals based on the disasters preparedness using the TOPSIS technique in western Iran. Hosp. Top. 2019, 97, 23-31. [CrossRef] [PubMed]

15. Ortiz-Barrios, M.A.; Aleman-Romero, B.A.; Rebolledo-Rudas, J.; Maldonado-Mestre, H.; Montes-Villa, L.; De Felice, F.; Petrillo, A. The analytic decision-making preference model to evaluate the disaster readiness in emergency departments: The ADT model. J. Multi-Criteria Decis. Anal. 2017, 24, 204-226. [CrossRef]

16. Roy, J.; Adhikary, K.; Kar, S.; Pamucar, D. A rough strength relational DEMATEL model for analysing the key success factors of hospital service quality. Decis. Mak. Appl. Manag. Eng. 2018, 1, 121-142. [CrossRef]

17. Sarkar, S. COVID-19 Susceptibility Mapping Using Multicriteria Evaluation. Disaster Med. Public Health Prep. 2020, 1-17. [CrossRef]

18. Sangiorgio, V.; Parisi, P. A multicriteria approach for risk assessment of Covid-19 in urban district lockdown. Saf. Sci. 2020, 130, 104862. [CrossRef]

19. Nardo, P.D.; Gentilotti, E.; Mazzaferri, F.; Cremonini, E.; Hansen, P.; Goossens, H.; Tacconelli, E. Multi-Criteria Decision Analysis to prioritize hospital admission of patients affected by COVID-19 in low-resource settings with hospital-bed shortage. Int. J. Infect. Dis. 2020. [CrossRef] 
20. Yildirim, F.S.; Sayan, M.; Sanlidag, T.; Uzun, B.; Uzun, D.; Ozsahin, I. A Clinical Decision Support System for the Treatment of COVID-19 with Multi-Criteria Decision-Making Techniques. JMIR Med. Inform. 2020. [CrossRef]

21. Biswas, T.K.; Das, M.C. Selection of the Barriers of Supply Chain Management in Indian Manufacturing Sectors Due to COVID-19 Impacts. Oper. Res. Eng. Sci. Theor. Appl. 2020, 3, 1-12.

22. Giordano, G.; Blanchini, F.; Bruno, R.; Colaneri, P.; Di Filippo, A.; Di Matteo, A.; Colaneri, M. Modelling the COVID-19 epidemic and implementation of population-wide interventions in Italy. Nat. Med. 2020, 26, 855-860. [CrossRef] [PubMed]

23. Kucharski, A.J.; Russell, T.W.; Diamond, C.; Liu, Y.; Edmunds, J.; Funk, S.; Eggo, R.M. Early dynamics of transmission and control of COVID-19: A mathematical modelling study. PRIME PubMed 2020, 20, 553-558. [CrossRef]

24. Diyaley, S.; Chakraborty, S. Optimization of multi-pass face milling parameters using metaheuristic algorithms. Facta Univ. Ser. Mech. Eng. 2019, 17, 365-383. [CrossRef]

25. Biswas, S.; Pamucar, D. Facility Location Selection for B-Schools in Indian Context: A Multi-Criteria Group Decision Based Analysis. Axioms 2020, 9, 77. [CrossRef]

26. Pamucar, D.; Deveci, M.; Canitez, F.; Lukovac, V. Selecting an Airport Ground Access Mode Using Novel Fuzzy LBWA-WASPAS-H Decision Making Model. Eng. Appl. Artif. Intell. 2020, 93, 103703. [CrossRef]

27. Deveci, M.; Özcan, E.; John, R.; Covrig, C.-F.; Pamucar, D. A Study on Offshore Wind Farm Siting Criteria Using a Novel Interval-valued Fuzzy-rough based Delphi Method. J. Environ. Manag. 2020, 270, 110916. [CrossRef] [PubMed]

28. Sinani, F.; Erceg, Z.; Vasiljević, M. An evaluation of a third-party logistics provider: The application of the rough Dombi-Hamy mean operator. Decis. Mak. Appl. Manag. Eng. 2020, 3, 92-107.

29. Petrovic, G.; Mihajlovic, J.; Cojbasic, Z.; Madic, M.; Marinkovic, D. Comparison of three fuzzy MCDM methods for solving the supplier selection problem. Facta Univ. Ser. Mech. Eng. 2019, 17, 455-469. [CrossRef]

30. Badi, I.; Shetwan, A.; Hemeda, A. A grey-based assessment model to evaluate health-care waste treatment alternatives in Libya. Oper. Res. Eng. Sci. Theory Appl. 2019, 2, 92-106. [CrossRef]

31. Durmić, E.; Stević, Ž.; Chatterjee, P.; Vasiljević, M.; Tomašević, M. Sustainable supplier selection using combined FUCOM-Rough SAW model. Rep. Mech. Eng. 2020, 1, 34-43. [CrossRef]

32. Pamucar, D. Normalized weighted Geometric Dombi Bonferoni Mean Operator with interval grey numbers: Application in multicriteria decision making. Rep. Mech. Eng. 2020, 1, 44-52. [CrossRef]

33. Pamucar, D.; Božanic, D.; Lukovac, V.; Komazec, N. Normalized weighted geometric Bonferroni mean operator of interval rough numbers-Application in interval rough DEMATEL-COPRAS. Facta Univ. Ser. Mech. Eng. 2018, 16, 171-191. [CrossRef]

34. Lazarevic, I. Using the ELECTRE MLO Multi-Criteria Decision-Making Method in Stepwise Benchmarking-Application in Higher Education. Oper. Res. Eng. Sci. Theory Appl. 2019, 2, 77-91. [CrossRef]

35. Pamucar, D.; Jankovic, A. The application of the hybrid interval rough weighted Power-Heronian operator in multi-criteria decision making. Oper. Res. Eng. Sci. Theory Appl. 2020, 3, 54-73. [CrossRef]

36. Zavadskas, E.K.; Kaklauskas, A.; Banaitis, A.; Kvederyte, N. Housing credit access model: The case for Lithuania. Eur. J. Oper. Res. 2004, 155, 335-352. [CrossRef]

37. Pamucar, D.; Vasin, L.J.; Lukovac, L. Selection of railway level crossings for investing in security equipment using hybrid DEMATEL-MARICA model. In Proceedings of the XVI International Scientific-Expert Conference on Railway, Railcon, Australia, 9-10 October 2014; pp. 89-92.

38. Pamucar, D.; Cirovic, G. The selection of transport and handling resources in logistics centres using Multi-Attributive Border Approximation area Comparison (MABAC). Expert Syst. Appl. 2015, 42, 3016-3028. [CrossRef]

39. Stevic, Z.; Pamucar, D.; Puska, A.; Chaterjee, P. Sustainable supplier selection in healthcare industries using a new MCDM method: Measurement of Alternatives and Ranking according to COmpromise Solution (MARCOS). Comput. Ind. Eng. 2020, 140, 106231. [CrossRef]

(C) 2020 by the authors. Licensee MDPI, Basel, Switzerland. This article is an open access article distributed under the terms and conditions of the Creative Commons Attribution (CC BY) license (http://creativecommons.org/licenses/by/4.0/). 